\title{
LOCALIZATIONS FOR CONSTRUCTION OF QUANTUM COSET SPACES
}

\author{
ZORAN ŠKODA \\ Department of Mathematics, Indiana University \\ Rawles Hall, Bloomington, IN 47405, U.S.A. \\ E-mail: zskoda@indiana.edu,zskoda@irb.hr
}

\begin{abstract}
Viewing comodule algebras as the noncommutative analogues of affine varieties with affine group actions, we propose rudiments of a localization approach to nonaffine Hopf algebraic quotients of noncommutative affine varieties corresponding to comodule algebras. After reviewing basic background on noncommutative localizations, we introduce localizations compatible with coactions. Coinvariants of these localized coactions give local information about quotients. We define Zariski locally trivial quantum group algebraic principal and associated bundles. Compatible localizations induce localizations on the categories of Hopf modules. Their interplay with the functor of taking coinvariants and its left adjoint is stressed out.

Using the localization approach, we construct a natural class of examples of quantum coset spaces, related to the quantum flag varieties of type A of other authors. Noncommutative Gauss decomposition via quasideterminants reveals a new structure in noncommutative matrix bialgebras. In the quantum case, calculations with quantum minors yield structure theorems.
\end{abstract}

Notation. The ground field is $\mathbf{k}$ and we assume it is of characteristic zero. If we deal just with one k-Hopf algebra, say $\mathcal{B}$, the comultiplication is $\Delta: \mathcal{B} \rightarrow \mathcal{B} \otimes \mathcal{B}$, unit map $\eta: \mathbf{k} \rightarrow \mathcal{B}$, counit $\epsilon: \mathcal{B} \rightarrow \mathbf{k}$, multiplication $\mu: \mathcal{B} \otimes \mathcal{B} \rightarrow \mathcal{B}$, and antipode (coinverse) $S: \mathcal{B} \rightarrow \mathcal{B}$. Warning: the letter $S$ often stands for a generic Ore set. We use [56, 49, 38, 74] Sweedler's notation $\Delta(h)=\sum h_{(1)} \otimes h_{(2)}$ with or without explicit summation sign, as well as its extension for coactions: $\rho(v)=\sum v_{(0)} \otimes v_{(1)}$, where the zero-component is in the comodule and the nonzero component(s) in the coalgebra. An entry symbol and name of a matrix will match, except for upper vs. lower case, e.g. $G=\left(g_{j}^{i}\right)$; and $G_{J}^{I}$ will be the submatrix with row multilabel $I=\left(i_{1}, \ldots, i_{k}\right)$ and column multilabel $J=\left(j_{1}, \ldots, j_{k}\right)$. As a rule, row labels are placed as superscripts and column labels as subscripts.

2000 Mathematics Subject Classification: Primary 14A22; Secondary 16W30, 14L30, 58B32.

Key words and phrases: coinvariants, Hopf algebra, quantum group, quantum principal bundle, localization, Ore set, matrix bialgebra, noncommutative Gauss decomposition.

The paper is in final form and no version of it will be published elsewhere. 
1. Introduction. There is an antiequivalence [32, 74] between the category of affine group schemes over $\mathbf{k}$ and the category of commutative Hopf algebras over $\mathbf{k}$. In the framework of affine group $\mathbf{k}$-varieties, the corresponding Hopf algebras are constructed as algebras of regular functions on the underlying group variety. Hence, taking the viewpoint of noncommutative geometry [8] we view Hopf algebras as noncommutative affine group varieties $[17,50,62]$.

Groups are useful as they describe the notion of symmetries: they act on spaces. A $B$-variety is an algebraic variety $E$ with a regular action $\nu: E \times B \rightarrow E$ of an algebraic group $B$. The Hopf algebra $\mathcal{O}(B)$ of regular functions on $B$ coacts on the algebra of regular functions on $\mathcal{O}(E)$ via the coaction $\rho: \mathcal{O}(E) \rightarrow \mathcal{O}(E) \otimes \mathcal{O}(B)$ given by the formula $\rho(e)(b)=\nu(e, b)$. This $\mathcal{O}(B)$-comodule structure on $\mathcal{O}(E)$ is compatible with the k-algebra structure on $\mathcal{O}(E)$ in the sense that the coaction is a k-algebra map, i.e. $\mathcal{O}(E)$ is an $\mathcal{O}(B)$-comodule algebra. Hence, in the noncommutative setup, comodule algebras are viewed as noncommutative $B$-varieties.

When we denote an algebra (Hopf algebra) by a caligraphic letter, say $\mathcal{E}$ (or $\mathcal{B}$ ) then the letter still suggests the underlying "variety", as in $\mathcal{E}=\mathcal{O}(E)$, however we replace $\mathcal{O}(E)$ by $\mathcal{E}$ precisely when we allow (though do not prescribe) noncommutative algebras.

Any function invariant on orbits of an action of a group on a set can be viewed as a function on the set of orbits. Invariant functions are coinvariants in the algebra of all functions with respect to the coaction of a Hopf algebra of functions on the group. This is mutatis mutadis true in various setups - finite sets, topological spaces, affine vs. nonaffine algebraic varieties, so we did not say it fully precisely. This is very important. Namely, if we reverse the question and ask whether we could describe the space of orbits (quotient space) by coinvariants, then, already in the commutative situation, the answer depends much on the category chosen, and even if we start with a nice-behaved category, the natural quotients should often be constructed in a larger category. One such phenomenon stems from the fact that observables on the quotient space have singularities. For example, if $G$ is an affine algebraic group and $B$ a closed algebraic subgroup, the quotient $G / B$ is, in general, not an affine variety, but it is always a quasiprojective variety [5]. In particular, it is often a projective variety where the only global regular functions are constants. However, there are many observables with the singularity locus of higher codimension and regular behavior away from the singularities. Hence it may be sufficient to introduce regular functions on Zariski open subsets of quotient spaces (complements of possible singularity loci). The idea of locally defined quotients is one of the starting points of the geometric invariant theory.

This survey gives an overview of efforts, at this point mainly of the present author, to access and use the local information on noncommutative quotients, mainly in the case of noncommutative coset spaces. The following are crucial observations in this programme:

- Noncommutative localizations are used to replace Zariski open subsets.

- Already in the commutative case, open sets in the quotient $E / B$ correspond (via the projection $E \rightarrow E / B$ ), not to arbitrary, but only to $B$-invariant open sets in $E$. To address this issue, in dual language, we introduce and study a notion of compatibility of a noncommutative localization with the coaction. The coaction naturally 
extends to a compatible localization. We obtain the localized coaction. Any compatible localization of a $\mathcal{B}$-comodule algebra $\mathcal{E}$ induces a localization functor from the category of those modules over $\mathcal{E}$ which are also $\mathcal{B}$-comodules in a compatible way (relative Hopf modules). They are analogues of $B$-equivariant (quasicoherent) sheaves on $E$.

- We study localized coinvariants, i.e. coinvariants for the localized coaction.

- In the case of noncommutative coset spaces, the existence of a covering by compatible localizations with large algebras of localized coinvariants justifies calling the latter charts in a coset space. Large is here in the sense of ability to perform descent, e.g. if the localization has an induced structure of a faithfully flat Hopf-Galois extension.

- Noncommutative Gauss decomposition for matrix bialgebras suggests natural candidates for (covers by) coaction compatible Cohn, and, in favorable cases, Ore localizations, such that the localized algebras can be trivialized as $\mathcal{B}$-bundles over the algebras of localized coinvariants. This provides a natural class of noncommutative candidates for coset spaces.

- Calculations with quasideterminants and, in the case of quantum groups, quantum minors, are useful techniques to study the above mentioned examples.

2. Commutative localizations (motivation). In (commutative) geometry localization appears as a means to

- pass from a space to an open subset of the space;

- pass to a different space reflecting only an infinitesimal neighborhood of a point or a subvariety.

In this paper we concentrate on the former flavor of localization. In the language of algebras, localizing can be done by introducing functions defined only locally. For affine varieties that means introducing inverses of those elements in the algebra whose zero set lies outside of the local set. There were also some attempts to use a localization for algebras of continuous functions [65], and that type of procedure may be useful for extensions of the present work to the operator-algebraic rather than algebraic setup.

The localized ring has a simpler structure than the original ring. Indeed, if $f$ is a generator of an ideal $I$ different from the whole ring $R$, then having $f^{-1}$ in the localized ring means that $f$ does not generate a proper ideal any more, as $f^{-1} f=1$. Hence, localization kills ideals. In particular it kills prime and maximal ideals and, as those correspond to points of schemes and varieties respectively, it removes some points from the space and the space gets smaller or "localized". For localization at a point we obtain a local ring.

If we introduce inverses of functions, we still know how to multiply them: pointwise. Noncommutative algebras are not algebras of functions on a genuine space consisting of points only, so we do not have a priori a fully satisfactory recipe for how to multiply the newly introduced inverses with other elements. There is an important case when such a recipe is known and elementary. That is the case of inverting all elements belonging to a given subset $S$ in a ring $R$ of special kind, called an Ore set. This is Ore localization. 
3. Ore localizations $[61,18,80]$. A semigroup $R$ with unit is called a monoid. A subset $S$ of a monoid $R$ is called multiplicative if $1 \in R$ and whenever $s_{1}, s_{2} \in S$ then $s_{1} s_{2} \in S$. Let $R$ be a (noncommutative) unital ring. We can also view it as a monoid with respect to multiplication. A multiplicative set $S \subset R \backslash\{0\}$ is called a left Ore set if the following left Ore conditions are satisfied:

- $\left(\forall s \in S \forall r \in R \exists s^{\prime} \in S \exists r^{\prime} \in R\right)\left(r^{\prime} s=s^{\prime} r\right)$ (left Ore condition proper)

- $(\forall n \in R \forall s \in S)\left((n s=0) \Rightarrow \exists s^{\prime} \in S\left(s^{\prime} n=0\right)\right)$ (left reversibility)

The left reversibility condition can be restated also as

$$
\left(\forall n_{1}, n_{2} \in R \forall s \in S\right)\left(\left(n_{1} s=n_{2} s\right) \Rightarrow \exists s^{\prime} \in S\left(s^{\prime} n_{1}=s^{\prime} n_{2}\right)\right),
$$

which has the advantage that it makes sense for arbitrary monoids, as well as, once the quantifiers are rewritten with care to appropriate source and target matching, also for groupoids, and categories. In the latter case we obtain a "left calculus of fractions" rather than a left Ore set, but the construction of localization (this time of a category) and accompanying proofs may proceed essentially the same way as for Ore sets.

For a left Ore set $S$ in a monoid $R$ define the monoid $S^{-1} R$ of left fractions as follows. As a set, $S^{-1} R:=S \times R / \sim$, where $\sim$ is the following equivalence relation:

$$
(s, r) \sim\left(s^{\prime}, r^{\prime}\right) \Leftrightarrow(\exists \tilde{s} \in S \exists \tilde{r} \in R)\left(\tilde{s} s^{\prime}=\tilde{r} s \text { and } \tilde{s} r^{\prime}=\tilde{r} r\right) .
$$

The equivalence class of $(s, r)$ is denoted $s^{-1} r$ and called a left fraction. The multiplication is defined by $s_{1}^{-1} r_{1} \cdot s_{2}^{-1} r_{2}=\left(\tilde{s} s_{1}\right)^{-1}\left(\tilde{r} r_{2}\right)$ where $\tilde{r} \in R, \tilde{s} \in S$ satisfy $\tilde{r} s_{2}=\tilde{s} r_{1}$ (one should think of this, though it is not yet formally justified at this point, as $\tilde{s}^{-1} \tilde{r}=r_{1} s_{2}^{-1}$, which enables one to put inverses next to one another and then the multiplication rule is obvious). If the monoid $R$ is a ring, then we can extend the addition to $S^{-1} R$ too. Suppose we are given two fractions with representatives $\left(s_{1}, r_{1}\right)$ and $\left(s_{2}, r_{2}\right)$. Then by the left Ore condition we find $\tilde{s} \in S, \tilde{r} \in R$ such that $\tilde{s} s_{1}=\tilde{r} s_{2}$. The sum is then defined

$$
s_{1}^{-1} r_{1}+s_{2}^{-1} r_{2}:=\left(\tilde{s} s_{1}\right)^{-1}\left(\tilde{s} r_{1}+\tilde{r} r_{2}\right)
$$

It is a long and at points tricky task to work out all the details of this definition. One has to show that $\sim$ is indeed an equivalence relation, that the operations are well defined, and that $S^{-1} R$ is indeed a ring. Even the commutativity of addition needs work. At the end one shows that $i=i_{S}: R \rightarrow S^{-1} R$ given by $i(r)=1^{-1} r$ is a homomorphism of rings, which is 1-1 iff the 2-sided ideal $I_{S}=\{n \in R \mid \exists s \in S$, sn $=0\}$ is zero.

If $S$ is left Ore, then we call the pair $\left(i, S^{-1} R\right)$ the left Ore localization of $R$ with respect to $S$. It has a universal property, namely, it is a universal object in the category $\mathcal{C}=\mathcal{C}(R, S)$ whose objects are pairs $(j, Y)$, where $j: R \rightarrow Y$ is a map into a ring $Y$ such that the image $j(S)$ of $S$ consists of units, and the morphisms $\alpha:(j, Y) \rightarrow\left(j^{\prime}, Y^{\prime}\right)$ are maps of rings $\alpha: Y \rightarrow Y^{\prime}$ such that $\alpha \circ j=j^{\prime}$. A universal object in $\mathcal{C}$ may exist when $S$ is not left Ore, for example when $S$ is right Ore and not left Ore. In fact, the universal object is a left Ore localization iff it lies in the full subcategory $\mathcal{C}^{l}$ of $\mathcal{C}$ whose objects $(j, Y)$ satisfy 2 additional conditions: $j(S)^{-1} j(R)=\left\{(j(s))^{-1} j(r) \mid s \in S, r \in R\right\}$ is a subring in $Y$ and $\operatorname{ker} j=I_{S}$. Hence $\left(i, S^{-1} R\right)$ is universal in $\mathcal{C}^{l}$, and that characterizes it, but the universality in $\mathcal{C}$, although not characteristic, appears to be more useful in practice. 
If $M$ is a left $R$-module then $S^{-1} M=S^{-1} R \otimes_{R} M$ is also a left module. This is the recipe for Ore localization of modules. The correspondence $Q_{S}: M \mapsto S^{-1} M$ is an exact endofunctor in the category of left $R$-modules, called the localization functor. For given $M$, the map $i_{S, M}: M \rightarrow S^{-1} M$ given by $m \mapsto 1 \otimes m$ is an $R$-module map, called the localization map.

Ore sets are relatively rare and also hard to single out. In practice, the Ore condition is checked on a suitable set of generators of a ring versus a suitable set of generators of the Ore set. One often uses induction arguments, recursively applying the Ore condition.

4. Ore vs. Gabriel localizations $[72,34]$. This section could be skipped on first reading as only few remarks in the paper depend on it. The modern viewpoint on localization as touched upon here is however essential for the current research in this area.

A lattice is a poset $(W, \succ)$ such that for any two elements $z_{1}, z_{2}$ the least upper bound $z_{1} \vee z_{2}$ and the greatest lower bound $z_{1} \wedge z_{2}$ exist. In other words, the binary operations of meet $\wedge$ and join $\vee$ are everywhere defined. A poset is bounded if it contains a maximum and a minimum element, which we denote 1 and 0 respectively. A filter in a bounded lattice $(W, \succ)$ is a subset $\mathcal{L} \subset W$ such that $1 \in \mathcal{L}, 0 \notin \mathcal{L},\left(z_{1}, z_{2} \in \mathcal{L} \Rightarrow z_{1} \wedge z_{2} \in \mathcal{L}\right)$ and $\left(z \in \mathcal{L}, z^{\prime} \succ z \Rightarrow z^{\prime} \in \mathcal{L}\right)$.

For any subset $w \subset R$, and any left ideal $J$, denote $(J: w)=\{z \in R \mid z w \in J\}$. It is also a left ideal. Let $I_{l} R$ be the preorder category of left ideals in a ring $R$ with respect to the inclusion preorder. It is a lattice. For the localization questions another partial order $\succ$ on $I_{l} R$ is sometimes better. Namely, $K \succ J$ iff either $J \subset K$ or there exists a finite subset $w \subset R$ such that $(J: w) \subset K$. Any filter in $\left(I_{l} R, \succ\right)$ is called a uniform filter.

For an Ore set $S \subset R$ consider $\mathcal{L}_{S}=\{J$ left ideal in $R \mid J \cap S \neq 0\} \subset I_{l} R$.

The left Ore condition implies at once that $\mathcal{L}_{S}$ can equivalently be defined by

$$
\mathcal{L}_{S}=\{J \text { left ideal in } R \mid \forall r(J: r) \cap S \neq 0\} .
$$

For any multiplicative subset $S \subset R$, not necessarily left Ore, formula (1) defines a Gabriel filter $\mathcal{L}_{S}$ of left ideals in $R$. It is a uniform filter.

To any Gabriel filter $\mathcal{L}$, one associates an endofunctor $\sigma_{\mathcal{L}}$ on the category of left $R$-modules by

$$
\sigma_{\mathcal{L}}(M)=\{m \in M \mid \exists J \in \mathcal{L}, J m=0\} .
$$

Equivalently, $\sigma_{\mathcal{L}}(M)=\lim _{J \in \mathcal{L}} \operatorname{Hom}_{R}(R / J, M)$. For example, if $\mathcal{L}=\mathcal{L}_{S}$ where $S$ is Ore, then $\sigma_{\mathcal{L}}(R)=I_{S}$ (see section 3$)$.

A subobject in a category is an equivalence class of monomorphisms. A functor $F$ is a subfunctor of a functor $G$ if $\operatorname{in}_{M}: F(M) \hookrightarrow G(M)$ is a subobject and the inclusions $\operatorname{in}_{M}: F(M) \hookrightarrow G(M)$ form a natural transformation of functors in : $F \rightarrow G$. Explicitly, $\operatorname{in}_{N} F(f)(F(M))=G(f)\left(\operatorname{in}_{M} F(M)\right)$ for $f: M \rightarrow N$.

If $\mathcal{A}$ is any Abelian category, then a subfunctor $\sigma$ of the identity (i.e. $\sigma(M) \subset M$ and $\sigma(f)(\sigma(M))=f(\sigma(M)))$ with the property $\sigma(M / \sigma(M))=0$ is called a preradical in $\mathcal{A}$. A radical is a left exact preradical. It follows that $\sigma_{\mathcal{L}}$ is an idempotent radical in the category of left $R$-modules, i.e. it is a radical and $\sigma_{\mathcal{L}}\left(\sigma_{\mathcal{L}}(M)\right)=\sigma_{\mathcal{L}}(M)$. 
To any Gabriel filter $\mathcal{L}$, one associates a localization endofunctor $Q_{\mathcal{L}}$ on the category of left modules by the formula

$$
Q_{\mathcal{L}}(M)=\lim _{J \in \mathcal{L}} \operatorname{Hom}_{R}\left(J, M / \sigma_{\mathcal{L}}(M)\right)
$$

It is not obvious that $Q_{\mathcal{L}}(M)$ is naturally a left $R$-module: the fact that $\mathcal{L}$ is a Gabriel filter plays a crucial role. Namely, given $f \in Q_{\mathcal{L}}(M)$, choose $J \in \mathcal{L}$ such that there is a $f_{J}$ in $\operatorname{Hom}_{R}\left(J, M / \sigma_{\mathcal{L}}(M)\right)$ representing $f$. For $r \in R$, the left ideal $(J: r) \in \mathcal{L}$, by the definition of a uniform filter, and the rule $x \mapsto f(x r)$ defines an element $(r f)_{(J: r)}$ in $\operatorname{Hom}_{R}\left((J: r), M / \sigma_{\mathcal{L}}(M)\right)$ representing the class of $r f$. This yields a well defined left action.

Left multiplication by an element $r \in R$ defines a class $[r] \in Q_{\mathcal{L}}(R)$. There is a unique ring structure on $Q_{\mathcal{L}}(R)$ such that the correspondence $i_{\mathcal{L}}: r \mapsto[r]$ becomes a ring homomorphism $i_{\mathcal{L}}: R \rightarrow Q_{\mathcal{L}}(R)$.

Not only every Gabriel filter defines an idempotent radical, every radical also defines a Gabriel filter by the rule

$$
\mathcal{L}_{\sigma}=\{J \subset R \mid \sigma(R / J)=R / J\}
$$

When we restrict to the idempotent radicals, then this rule gives a bijection between the idempotent radicals and Gabriel filters.

Though it does not behave as nicely as Ore localization does, scarcity of Ore sets makes Gabriel localization attractive and it is widely used. Moreover, this more general class of localizations can be phrased fully in the language of the Abelian category of left $R$-modules, and it generalizes to other Abelian categories with some good properties. A common generality in which this is studied are Grothendieck categories. A Grothendieck category $\mathcal{A}$ is an Abelian category which is cocomplete (small inductive limits always exist $[4,47,90]$ ), where filtered limits are exact, and which has a generator (an object $G$ in $\mathcal{A}$ such that $C \mapsto \operatorname{Hom}_{\mathcal{C}}(C, G)$ is a faithful functor), for details cf. [34, 64, 83, 90, 81]. Such categories are a natural place to study noncommutative algebraic geometry beyond the affine and projective cases $[71,81,59]$.

A thick subcategory of an Abelian category $\mathcal{A}$ is a replete (= full and closed under isomorphisms) subcategory $\mathcal{T}$ of $\mathcal{A}$ which is closed under extensions, subobjects and quotients. In other words, an object $M^{\prime}$ in a short sequence $0 \rightarrow M \rightarrow M^{\prime} \rightarrow M^{\prime \prime} \rightarrow 0$ in $\mathcal{A}$ belongs to $\mathcal{T}$ iff $M$ and $M^{\prime \prime}$ do. Localization at thick subcategories is a common framework in noncommutative algebraic geometry [71, 68]. Starting from a pair $(\mathcal{A}, \mathcal{T})$ where $\mathcal{A}$ is Abelian and $\mathcal{T}$ is thick, one forms a (Serre) quotient category $[4,19,20,34,64$, 68]. As objects one takes the objects of the original category, but in addition to the original morphisms one adds to the class of morphisms the formal inverses of those morphisms $f$ for which both Ker $f$ and Coker $f$ are in $\mathcal{T}$. A thick subcategory is called a (Serre) localizing subcategory if the morphisms which are invertible in the quotient category are exactly those for which Ker $f$ and Coker $f$ are both in $\mathcal{T}$. Hence, more than one thick subcategory may give the same quotient category, and that ambiguity is removed if we consider the corresponding localizing subcategories instead.

For any idempotent radical $\sigma$ in $\mathcal{A}$, define the class $\mathcal{T}_{\sigma}$ of $\sigma$-torsion objects and the class $\mathcal{F}_{\sigma}$ of $\sigma$-torsion free objects by 


$$
\mathcal{T}_{\sigma}=\{M \in \mathrm{Ob} \mathcal{A} \mid \sigma(M)=M\}, \quad \mathcal{F}_{\sigma}=\{M \in \mathrm{Ob} \mathcal{A} \mid \sigma(M)=0\}
$$

The pair $\left(\mathcal{T}_{\sigma}, \mathcal{F}_{\sigma}\right)$ is an example of a torsion theory and $\mathcal{T}_{\sigma}$ is a thick subcategory of $\mathcal{A}$. A torsion theory $[4,34]$ in an Abelian category $\mathcal{A}$ is a pair $(\mathcal{T}, \mathcal{F})$ of replete subcategories of $\mathcal{A}$ such that every morphism $T \rightarrow F$, where $T$ is an object in $\mathcal{T}$ and $F$ an object in $\mathcal{F}$, is a zero morphism; and such that every object $A$ in $\mathcal{A}$ can be put into an exact sequence $0 \rightarrow T \rightarrow A \rightarrow F \rightarrow 0$ where $T$ is an object in $\mathcal{T}$ and $F$ an object in $\mathcal{F}$. Localizations in Abelian categories, and categories of modules in particular, are often conveniently described in terms of torsion theories.

A torsion theory is hereditary if every submodule of a torsion module is also torsion. Torsion theories correspond to idempotent preradicals. Hereditary torsion theories correspond to Gabriel localizations, which in turn correspond to idempotent radicals.

5. Covers via localizations $[72,69,34,80]$. For a moment, we take the most general view $[69,20,4]$ that a localization is a functor $Q^{*}: \mathcal{C} \rightarrow \mathcal{C}^{\prime}$ which is universal [20] with respect to inverting some class $\Sigma$ of morphisms in $\mathcal{C}$. A functor $Q^{*}$ between Abelian categories will be called continuous if it has a right adjoint, say $Q_{*}$, and flat if $Q^{*}$ is, in addition, exact. A characterization of a localization functor is [20] that it has a fully faithful right adjoint. A family $\mathcal{V}=\left(Q_{\lambda}^{*}: \mathcal{C} \rightarrow \mathcal{C}_{\lambda}\right)_{\lambda \in \Lambda}$ of flat functors covers $\mathcal{C}$ if it is conservative, i.e.

$$
\forall f \in \operatorname{Mor} \mathcal{C}\left(\left((\forall \lambda) Q_{\lambda}^{*}(f) \text { is invertible }\right) \Rightarrow f \text { is invertible }\right) .
$$

We are interested here in covers by localizations only, but we expect more general flat covers to play a role in future extensions of this work, as they do in commutative algebraic geometry. Flat covers by localizations are called by A. Rosenberg [69] Zariski covers.

Let us now specialize to the category of left $R$-modules. We would like to carry further the picture that localizations correspond to certain open subsets. In addition to covers, one would like to have "intersections". A newcomer to localization should be warned, however, of pitfalls here.

In the case of left Ore localizations $S^{-1} R$ and $T^{-1} R$, the natural candidate for intersection is the localization at the set $S \vee T$ multiplicatively generated by $S$ and $T$. It is automatically Ore, hence $(S \vee T)^{-1} R$ is a ring as usual. The set $S T$ of products st, $s \in S, t \in T$ is, in general, not multiplicative, but it does satisfy the left Ore condition.

As $S^{-1} R$ is an $R$-module, one can always introduce $T^{-1} S^{-1} R$ as an $R$-module, by applying the localization at $S$ first, and the localization at $T$ after that. $T^{-1} S^{-1} R$ is not necesarily a ring via Ore construction, as inverting $T$ (more precisely, $i_{S}(T)$ ) in $S^{-1} R$ by Ore method asks for $i_{S}(T)$ to be left Ore in $S^{-1} R$, which is not true in general, and replacing left Ore sets by 2 -sided Ore sets does not help. To get a feeling for this phenomenon write down the Ore condition for $i_{S}(T)$ in $S^{-1} R$ and notice that there is more to check than the original Ore conditions for $T$ in $R$ say. Similarly, we can consider $S^{-1} T^{-1} R$ and even $T^{-1} S^{-1} T^{-1} R$ etc.

$(S \vee T)^{-1} R$ is isomorphic to $T^{-1} S^{-1} R$ as an $R$-module precisely when $i_{S}(T)$ is left Ore in $S^{-1} R$, hence, by symmetry, iff $i_{T}(S)$ is left Ore in $T^{-1} R$. If this is true, which is rare in the noncommutative case, we say that $S$ and $T$ are mutually compatible left Ore sets $[34,89]$ (not to confuse with the compatibility with coaction which is a central topic 
in this paper). Hence, if $S$ and $T$ are compatible, the module $T^{-1} S^{-1} R$ has a natural ring structure (characterized also by the requirement that $\left(i_{T}, T^{-1} S^{-1} R\right)$ is an object in $\left.\mathcal{C}\left(S^{-1} R, T\right)\right)$. Following A. Rosenberg, we call a cover semiseparated if the localizations are pairwise compatible (this makes sense for localizations more general than Ore).

As $S \vee T$ is a bigger set than $S T$, we loose some information (kill more ideals) using localization at $S \vee T$ instead of the consecutive localizations at $S$ and $T$. More concretely, if we view $\vee$ as an operation of taking "intersection of open sets" and if a ring covered by localizations could be considered as a "union" of such, then we face the fact that "intersection" is not distributive with respect to "union": $\left\{\left(S \vee T_{\lambda}\right)^{-1} R\right\}_{\lambda \in \Lambda}$ does not necesarily cover $S^{-1} R$ if $\left\{T_{\lambda}^{-1} R\right\}_{\lambda \in \Lambda}$ covers $R$.

However, there is a positive result which puts us in business:

Globalization Lemma. Suppose a finite family of Ore localizations $\left\{T_{\lambda}^{-1} R\right\}_{\lambda \in \Lambda}$ covers $R$. Then for every left $R$-module $M$ the sequence

$$
0 \rightarrow M \rightarrow \prod_{\lambda \in \Lambda} T_{\lambda}^{-1} M \rightarrow \prod_{(\mu, \nu) \in \Lambda \times \Lambda} T_{\mu}^{-1} T_{\nu}^{-1} M
$$

is exact, where the first morphism is $m \mapsto \prod i_{\lambda}(m)$ and the second is

$$
\prod_{\lambda} m_{\lambda} \mapsto \prod_{(\mu, \nu)}\left(i_{\mu, \nu}^{\mu}\left(m_{\mu}\right)-i_{\mu, \nu}^{\nu}\left(m_{\nu}\right)\right) .
$$

Here $i_{\lambda}$ is the localization map and $i_{\mu, \nu}^{\mu}$ is the natural map from $T_{\mu}^{-1} M$ to $T_{\mu}^{-1} T_{\nu}^{-1} M$, hence the lower indices for $i$ denote the target and the order matters.

This statement has been generalizated to Gabriel filters [72], cf. also [34]. This may be derived from the application of Barr-Beck's theorem in this setup.

The meaning of the globalization lemma is that every module can be reconstructed by gluing from its localizations, provided the overlaps in successive localizations in both orders are taken simultaneously into account, as it is in the general picture of flat descent, and this principle extends to triple etc. localizations. Two systematic methods to use this basic fact about covers have been proposed.

The $1^{\text {st }}$ method, proposed by F. van Oystaeyen and L. Willaert, is to organize covers of some considered type into a noncommutative (analogue of a) Grothendieck topology [91, $92,34,59]$, noncommutativity referring to the fact discussed above that the order of taking successive localizations matters. The notions of a sheaf and a quasicoherent sheaf are then directly defined in analogy to the commutative situation [89]. Most of the work in this direction is focused on the case of $\mathbb{Z}_{>0}$-graded Noetherian algebras for which a nontrivial finite cover by nontrivial Ore sets exists, so called schematic algebras [91, 89].

The $2^{\text {nd }}$ method centers on a comonad [69] associated to a given flat cover, to place it transparently in the general picture of flat descent $[3,12,28,70]$. Then one associates a cosimplicial object [69] to the comonad. When applying various functors to this construction the exactness properties of the functors and of the comonad play a decisive role; the description of objects obtained by gluing local data depends on the applicability of Barr-Beck's theorem [3, 4, 2, 40, 47, 48]. In particular, this is suitable for descent-type questions, and the construction of quotients can also be understood that way. 
We say that a family $T_{\mu}^{-1} R$ of Ore localizations naively covers a ring $R$ if

$$
0 \rightarrow R \rightarrow \prod_{\lambda \in \Lambda} T_{\lambda}^{-1} R \rightarrow \prod_{\mu<\nu}\left(T_{\mu} \cup T_{\nu}\right)^{-1} R
$$

is exact. Here the second map is

$$
\prod_{\lambda} m_{\lambda} \mapsto \prod_{\mu<\nu}\left(i_{\mu, \nu}\left(m_{\mu}\right)-i_{\nu, \mu}\left(m_{\nu}\right)\right)
$$

where $i_{\mu, \nu}$ (omitted upper indices!) is the map $i_{\mu, \nu}: T_{\mu}^{-1} R \rightarrow\left(T_{\mu} \cup T_{\nu}\right)^{-1} R$. Every naive cover is a cover, and every semiseparated cover is a naive cover. For the case of two localizations only, naive covers coincide with semiseparated covers. Covers appear more naturally than naive covers do, and having a naive (but nonsemiseparated) cover does not guarantee much more than a cover can do.

6. Trivial principal bundles. Given an affine algebraic group $B$ with a regular right action $\nu: E \times B \rightarrow E$ on an affine variety $E$, define a linear map

$$
\rho \equiv \rho_{\nu}: \mathcal{O}(E) \rightarrow \mathcal{O}(E \times B) \cong \mathcal{O}(E) \otimes \mathcal{O}(B) \text { by } \rho_{\nu}(f)(e, b)=f(\nu(e, b))
$$

The map $\rho_{\nu}$ is a right $\mathcal{O}(B)$-coaction and an algebra map. In the noncommutative case $\mathcal{O}(B)$ will be generalized to an arbitrary Hopf algebra $\mathcal{B}$, and $E$ to any $\mathcal{O}(B)$-comodule algebra $\mathcal{E}$. Any left (right) comodule $\mathcal{E}$ over a bialgebra $\mathcal{B}$ such that $\mathcal{E}$ is an associative algebra and the coaction is a homomorphism of algebras is called a left (right) $\mathcal{B}$-comodule algebra.

For a set $E$ with a right $B$-action define

$$
E \times{ }_{B} E=\left\{\left(e_{1}, e_{2}\right) \in E \times E \mid \exists b \in B, e_{1} b=e_{2}\right\} \subset E \times E .
$$

If $E$ is a topological space then $E \times{ }_{B} E$ inherits a subspace topology from $E \times E$. The action of $B$ is free if for every pair $\left(e_{1}, e_{2}\right) \in E \times_{B} E$ there is a unique $b$ with $e_{1} b=e_{2}$. Then the rule $\tau:\left(e_{1}, e_{2}\right) \mapsto b$ defines a map of sets $\tau: E \times_{B} E \rightarrow E$. If $B$ is a topological group a principal $B$-bundle is a topological space $E$ with a free right $B$-action such that $\tau$ is a continuous map. In addition, a local triviality condition is usually required.

For algebraic varieties continuous maps are replaced by regular maps.

A principal $B$-bundle is trivial if there is a section $t: X \rightarrow E$ of the projection $p: E \rightarrow X$, i.e. a continuous map such that $p \circ t=\mathrm{id}_{X}$. Let $t$ be a section, $p^{-1}(x)$ some fiber, and $f$ a continuous function on $B$. Then the formula

$$
\gamma_{t}(f)(e)=f(\tau(t(p(e)), e))
$$

defines a continuous function $\gamma_{t}(f)$ on $E$. In the algebraic case, the map

$$
\gamma_{t}: \mathcal{B} \rightarrow \mathcal{E}, \quad f \mapsto \gamma_{t}(f)
$$

defines a map of commutative $\mathcal{B}$-comodule algebras, where $\mathcal{B}=\mathcal{O}(B)$ etc.

To prepare for the study of locally trivial principal bundles we now introduce certain free and smash products, and a notion of compatibility.

Let $\mathcal{B}$ be a k-bialgebra and $\left(M_{\alpha}, \rho_{\alpha}\right)$ a family of $\mathcal{B}$-comodules. A family of k-linear maps $f_{\alpha}: M_{\alpha} \rightarrow A$ where $A$ is a fixed algebra is called $\left\{\rho_{\alpha}\right\}$-compatible iff there is a 
unique coaction $\rho_{A}$ on $A$ making $A$ a $\mathcal{B}$-comodule algebra and for each $\alpha$ the diagram

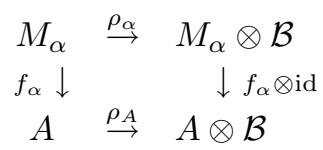

commutes.

EXAmPLES. 1. Compatible localization maps, cf. 8.1.

2. Given a right $\mathcal{B}$-comodule $(V, \rho)$, the inclusion $V \hookrightarrow T(V)$ is $\rho$-compatible.

3. Let $\left(A_{1}, \rho_{1}\right),\left(A_{2}, \rho_{2}\right), \ldots,\left(A_{n}, \rho_{n}\right)$ be a finite sequence of right $\mathcal{B}$-comodule algebras. The family of natural inclusions $i_{j}: A_{j} \rightarrow A_{1} \otimes A_{2} \otimes \ldots \otimes A_{n}$ is $\left\{\rho_{j}\right\}$-compatible.

4. A free product of a family of associative algebras $\left\{A_{j}\right\}_{j \in \mathcal{J}}$ is $T\left(\oplus_{j \in \mathcal{J}} A_{j}\right)$ modulo an ideal generated by all expressions of the form $a \otimes a^{\prime}-a a^{\prime}$ where both $a, a^{\prime}$ belong to $A_{j}$ with the same $j$ If all $A_{j}$ are $\mathcal{B}$-comodule algebras, then combining Examples 2 and 3 and this description conclude that the family of natural inclusions $i_{j}: A_{j} \hookrightarrow \star_{j \in J} A_{j}$ is $\left\{\rho_{j}\right\}$-compatible.

4a. In particular, consider an algebra $U$ with the trivial $\mathcal{B}$-coaction and $\mathcal{B}$ self-coacting by comultiplication. By compatibility, $U \star \mathcal{B}$ becomes a $\mathcal{B}$-comodule algebra.

Let $U$ be an algebra and $\mathcal{B}$ a Hopf algebra. Define the category $\mathcal{C}=\mathcal{C}(U, \mathcal{B})$ of higher smash products of $U$ and $\mathcal{B}$ as follows. An object in $\mathcal{C}$ is a triple $(A, \iota, \gamma)$ where $A$ is a $\mathcal{B}$-comodule algebra, $\iota: U \hookrightarrow A^{\text {co } \mathcal{B}}$ is a 1 -1 algebra map, and $\gamma: \mathcal{B} \rightarrow A$ is a $\mathcal{B}$-comodule algebra map such that $A$ is generated by $\iota(U)$ and $\gamma(\mathcal{B})$. A morphism in $\mathcal{C}$ from $(A, \iota, \gamma)$ into $\left(A^{\prime}, \iota^{\prime}, \gamma^{\prime}\right)$ is a map $f: A \rightarrow A^{\prime}$ of $\mathcal{B}$-comodule algebras such that $\iota^{\prime}=\iota \circ f$ and $\gamma^{\prime}=\gamma \circ f$.

For every object in $\mathcal{C}$, one defines a map

$$
\triangleright: \mathcal{B} \otimes A \rightarrow A, \quad b \triangleright a=\sum \gamma\left(b_{(1)}\right) a \gamma\left(S b_{(2)}\right) .
$$

This is an algebra action making $A$ a $\mathcal{B}$-module algebra (i.e. $b \triangleright 1_{A}=1_{A}$ and $b \triangleright\left(a a^{\prime}\right)=$ $\left.\sum\left(b_{(1)} \triangleright a\right)\left(b_{(2)} \triangleright a^{\prime}\right)\right)$, and $A^{\text {co } \mathcal{B}}$ a $\mathcal{B}$-submodule subalgebra. Moreover, $A^{\text {co } \mathcal{B}}$ is the smallest $\mathcal{B}$-stable (i.e. $\triangleright$-invariant) subalgebra of $A$ containing $U$. It follows that $A$ is isomorphic, as a comodule algebra, to the (ordinary) smash product $A^{\mathrm{co}} \mathcal{B}_{\sharp \mathcal{B}}$ for that action on $A^{\text {co } \mathcal{B}}$, i.e. the tensor product $A^{\text {co } \mathcal{B}} \otimes \mathcal{B}$ with product rule $(v \otimes b)\left(v^{\prime} \otimes b^{\prime}\right)=$ $v\left(b_{(1)} \triangleright v^{\prime}\right) \otimes b_{(2)} b^{\prime}$ and $\mathcal{B}$-coaction id $\otimes \Delta_{\mathcal{B}}$.

The free product $U \star \mathcal{B}$ with the induced $\mathcal{B}$-coaction (cf. Example $4 \mathrm{a}$, above) and the natural inclusions $\iota_{U}: U \hookrightarrow U \star \mathcal{B}$ and $\gamma: \mathcal{B} \hookrightarrow U \star \mathcal{B}$, form a universal object in $\mathcal{C}$. Any ordinary smash product $U \sharp \mathcal{B}$ in $\mathcal{C}(U, \mathcal{B})$ is locally terminal in the sense that any morphism in $\mathcal{C}$ with source $U \sharp \mathcal{B}$ is an isomorphism in $\mathcal{C}$, and that for any object $C$ there is at most one morphism from $C$ to $U \sharp \mathcal{B}$. All locally terminal objects in $\mathcal{C}$ are of that type. Isomorphism classes in $\mathcal{C}$ of ordinary smash products (with the same $U$ ) are distinguished by the action $\triangleright$. For every given $\mathcal{B}$-module algebra action on $U$, there is a unique isomorphism class of locally terminal objects in $\mathcal{C}$ such that $\iota_{U}$ is a map of $\mathcal{B}$-modules.

Finally, $\mathcal{C}$ is an umbrella category, i.e. it has a universal initial object and a class $\theta$ of locally terminal objects, and for every object $C$ in $\mathcal{C}$ there is at least one $T \in \theta$ with a (unique) morphism $C \rightarrow T$ in $\mathcal{C}$. 
A trivial quantum principal $\mathcal{B}$-bundle is an object in $\mathcal{C}(U, \mathcal{B})$ for some algebra $U$. Notice that for the same $\mathcal{B}$ and the same base space $U$ there is more than one trivial bundle, as the action $\triangleright$ can be different. In our point of view even for a fixed total space we allow different $i(U) \subset A^{\mathrm{co} \mathcal{B}}$ as long as $\iota(U)$ and $\gamma(\mathcal{B})$ generate $A$. Namely a natural candidate for the quantum base space is sometimes smaller than the whole algebra of coinvariants, and the latter appears with the help of the action in the total space (so it is not fully "base").

7. Commutative local triviality and torsors. For algebraic principal bundles, local triviality is considered with respect to one of the several standard topologies for schemes. Local triviality in Zariski topology is the strongest requirement, and the local trivialities in étale, fppf, fpqc topology are weaker, in that order [14,53]. A principal bundle locally trivial in étale topology is often called a torsor. If the orbit space is denoted by $X$, one can replace $B$ by a trivial $B$-bundle $\mathbf{B}$ over $X$ (i.e. by the product $B \times X$ ). Then $\mathbf{B}$ is a (relative) group scheme over $X$. More generally, consider any group scheme $\mathbf{B}$ over $X$ (topological analogue: a bundle of groups over $X$ ) acting upon $X$ in the category of schemes over $X$. Descent theory implies [53] that local triviality of $E$ over $X$ in flat topology is equivalent to the requirement that $E$ is faithfully flat and locally of finite type over $X$ and that

$$
(e, b) \mapsto(e, e b): E \times_{X} \mathbf{B} \rightarrow E \times_{X} E
$$

is an isomorphism. If $E, X$ and $B$ are affine, then we can dualize (4) by taking global sections of the structure sheaf [14]. In that case $\mathcal{X}=\Gamma \mathcal{O}_{X}$ is the ring of $\Gamma \mathcal{O}_{B}$-coinvariants in $\mathcal{E}=\Gamma \mathcal{O}_{E}$. Then, if (4) is isomorphism, it follows [14] that the map

$$
\mathcal{E} \otimes_{\mathcal{X}} \mathcal{E} \rightarrow \mathcal{E} \otimes_{\mathbf{k}} \mathcal{B}, \quad e \otimes_{\mathcal{X}} e^{\prime} \mapsto\left(e \otimes_{\mathbf{k}} 1_{\mathcal{B}}\right) \rho\left(e^{\prime}\right)
$$

is bijective. An extension of any algebra $\mathcal{X}$ by a Hopf algebra $\mathcal{B}$ is a $\mathcal{B}$-comodule algebra $\mathcal{E}$ such that $\mathcal{X}$ equals to the ring $\mathcal{E}^{\mathrm{co} \mathcal{B}}$ of coinvariants in $\mathcal{E}$. An extension is Hopf-Galois iff the map (5) is bijective. An extension is cleft if there is a convolution-invertible map of $\mathcal{B}$-comodules $\gamma: \mathcal{B} \rightarrow \mathcal{E}$ (cf. (3)). For any cleft extension there is a $\mathbf{k}$-linear map

$$
\triangleright: \mathcal{B} \otimes \mathcal{X} \rightarrow \mathcal{X}, \quad b \triangleright u=\sum \gamma\left(b_{(1)}\right) u \gamma^{-1}\left(b_{(2)}\right),
$$

as it is direct to check that the right-hand side is a $\mathcal{B}$-coinvariant. The map $\triangleright$ measures $\mathcal{X}$, i.e. $b \triangleright 1=1$ and $b \triangleright(u v)=\sum\left(b_{(1)} \triangleright u\right)\left(b_{(2)} \triangleright v\right)$. Cleft extensions are (a special case of) Hopf-Galois extensions. $\left(\mathcal{E}, \mathrm{id}_{\mathcal{E}^{\mathrm{co \mathcal {B }}}}, \gamma\right)$ is a trivial quantum principal $\mathcal{B}$-bundle over $\mathcal{E}^{\operatorname{co} B}$ if $\gamma$ is also an algebra map. In that case, convolution invertibility of $\gamma$ comes for free as $\gamma^{-1}=\gamma \circ S$.

\section{Localized coinvariants}

8.1. Compatibility. Suppose we are given a bialgebra $\mathcal{B}$ and a right $\mathcal{B}$-comodule algebra $\mathcal{E}$. An Ore set $T$ and a localization $i_{T}: \mathcal{E} \rightarrow T^{-1} \mathcal{E}$ are compatible with a coaction $\rho: \mathcal{E} \rightarrow \mathcal{E} \otimes \mathcal{B}$ if there exists a unique map

$$
\rho_{T}: T^{-1} \mathcal{E} \rightarrow T^{-1} \mathcal{E} \otimes \mathcal{B}
$$


which makes $\left(T^{-1} \mathcal{E}, \rho_{T}\right)$ into a $\mathcal{B}$-comodule algebra and the following diagram commutes:

$$
\begin{array}{ccc}
\mathcal{E} & \stackrel{\rho}{\rightarrow} & \mathcal{E} \otimes \mathcal{B} \\
i_{T} \downarrow & & i_{T} \otimes \mathrm{id} \downarrow \\
T^{-1} \mathcal{E} & \stackrel{\rho_{T}}{\rightarrow} & T^{-1} \mathcal{E} \otimes \mathcal{B},
\end{array}
$$

i.e. $\rho_{T} \circ i_{T}=\left(i_{T} \otimes \mathrm{id}\right) \circ \rho$.

In other words, Ore localization is $\rho$-compatible iff there is a unique $\mathcal{B}$-comodule algebra structure on the localized algebra such that the localization map is an intertwiner.

This definition is still appropriate for those more general (than Ore) localizations for which $Q(R)$ is still naturally a ring with homomorphism $i: R \rightarrow Q(R)$, and the composition of functors $Q^{*} i_{*}$ (where $i_{*}$ is the restriction of scalars functor from $Q(R)$ modules to $R$-modules) is an equivalence between the category of $Q(R)$-modules and the localized category. (Only) such localization functors satisfy $Q(M)=Q(R) \otimes_{R} M$ for all $M$. They are called perfect localizations [34, 83]. A Gabriel localization is perfect iff $Q=Q_{*} Q^{*}$ is an exact endofunctor. For even more general cases, one may redefine the $\rho$-compatibility in the language of Hopf module categories.

Classically we think of $\rho$-compatibility as the condition that the corresponding Zariski open set is $B$-invariant, i.e. a union of $B$-orbits.

Any $\rho$-compatible Ore localization $i_{S}: \mathcal{E} \rightarrow S^{-1} \mathcal{E}$ of a cleft $\mathcal{B}$-extension $\mathcal{E}$ is cleft. The section is $\gamma_{S}=i_{S} \circ \gamma$. If $\gamma$ is an algebra map, so is $\gamma_{S}$. This is forced by the very definition of the $\rho$-compatibility.

8.2. Practical criterium of compatibility. The localization $\mathcal{E}\left[T^{-1}\right]$ is $\rho_{T}$-compatible iff for each $t \in T$, the element $\left(\left(i_{T} \otimes \mathrm{id}\right) \circ \rho\right)(t)$ is invertible in the algebra $T^{-1} \mathcal{E} \otimes \mathcal{B}$.

The proof is elementary, cf. [76, 77].

8.3. Localized coinvariants $[76,77]$. Let $T$ be a $\rho$-compatible right Ore set in a right $\mathcal{B}$ comodule algebra $(\mathcal{E}, \rho)$. By compatibility there is a uniquely defined localized $\mathcal{B}$-coaction $\rho_{T}$ on $\mathcal{E}_{T}=T^{-1} \mathcal{E}$. We define the algebra of $T$-localized right $\rho$-coinvariants in $\mathcal{E}$ to be the algebra of $\rho_{T}$-coinvariants in $\mathcal{E}_{T}$ :

$$
\mathcal{E}_{T}^{\mathrm{co} \mathcal{B}}=\left\{y \in \mathcal{E}_{T} \mid \rho_{T} y=y \otimes 1\right\} .
$$

8.4. Nested localizations $[76,77]$. Let $S \subset T \subset \mathcal{E}$ be an inclusion of Ore subsets in $\mathcal{E}$, both compatible with $\mathcal{B}$-coaction $\rho$.

(i) The square diagram involving localized coactions commutes:

$$
\begin{array}{ccc}
S^{-1} \mathcal{E} & \stackrel{\rho_{S}}{\rightarrow} & S^{-1} \mathcal{E} \otimes \mathcal{B} \\
i_{T}^{S} \downarrow & & \downarrow i_{T}^{S} \otimes \mathrm{id} \\
T^{-1} \mathcal{E} & \stackrel{\rho_{T}}{\rightarrow} & T^{-1} \mathcal{E} \otimes \mathcal{B}
\end{array}
$$

In other words, the natural maps $i_{T}^{S}$ between localizations are intertwiners.

(ii) The natural map $i_{T}^{S}$ between the localizations maps the (sub)algebra of $S$-localized coinvariants into the (sub)algebra of $T$-localized coinvariants:

$$
i_{T}^{S}\left(\left(S^{-1} \mathcal{E}\right)^{\mathrm{co} \mathcal{B}}\right) \subset\left(T^{-1} \mathcal{E}\right)^{\mathrm{co} \mathcal{B}}
$$

8.5. Compatibility and Hopf modules. Given a right $\mathcal{B}$-comodule algebra $\mathcal{E}$, (relative) $(\mathcal{E}, \mathcal{B})$-Hopf modules, or simply dimodules, are the objects of the category ${ }_{\mathcal{E}} \mathcal{M}^{\mathcal{B}}$ formed 
by left $\mathcal{E}$-modules $M$ which are also right $\mathcal{B}$-comodules, with compatibility

$$
\sum e_{(0)} m_{(0)} \otimes_{\mathbf{k}} e_{(1)} m_{(1)}=\sum(e m)_{(0)} \otimes_{\mathbf{k}}(e m)_{(1)} .
$$

For any $M \in{ }_{\mathcal{E}} \mathcal{M}^{\mathcal{B}}$ one defines the $\mathcal{B}$-coaction $\rho^{\prime}$ on the tensor product $S^{-1} \mathcal{E} \otimes_{\mathbf{k}} M$ over the ground field $\mathbf{k}$ :

$$
\begin{aligned}
& \rho^{\prime}: S^{-1} \mathcal{E} \otimes_{\mathbf{k}} M \rightarrow S^{-1} \mathcal{E} \otimes_{\mathbf{k}} M \otimes_{\mathbf{k}} \mathcal{B}, \\
& \rho^{\prime}\left(x \otimes_{\mathbf{k}} m\right)=\sum\left(x_{(0)} \otimes_{\mathbf{k}} m_{(0)}\right) \otimes_{\mathbf{k}} x_{(1)} m_{(1)} .
\end{aligned}
$$

This coaction factors to the $\mathcal{B}$-coaction $\rho_{M}$ on the $S^{-1} M$ which is the tensor product of the same factors but now over $\mathcal{E}$ :

$$
\rho_{S^{-1} M}\left(x \otimes_{\mathcal{E}} m\right)=\sum\left(x_{(0)} \otimes_{\mathcal{E}} m_{(0)}\right) \otimes_{\mathbf{k}} x_{(1)} m_{(1)},
$$

i.e. we have the commutative diagram

$$
\begin{array}{ccc}
S^{-1} \mathcal{E} \otimes_{\mathbf{k}} M & \stackrel{\rho^{\prime}}{\rightarrow} & \left(S^{-1} \mathcal{E} \otimes_{\mathbf{k}} M\right) \otimes_{\mathbf{k}} \mathcal{B} \\
\downarrow \text { pr } & & \downarrow \text { pr } \otimes_{\mathbf{k}} \text { id } \\
S^{-1} M=S^{-1} \mathcal{E} \otimes_{\mathcal{E}} M & \stackrel{\rho_{S^{-1} M}}{\rightarrow} & \left(S^{-1} \mathcal{E} \otimes_{\mathcal{E}} M\right) \otimes_{\mathbf{k}} \mathcal{B}
\end{array}
$$

where the vertical maps are the natural projections. The bottom map is well-defined thanks to the compatibility of $\mathcal{E}$-module and $\mathcal{B}$-comodule structure on $M$ :

$$
\begin{aligned}
\sum\left((y e)_{(0)} \otimes_{\mathcal{E}} n_{(0)}\right) \otimes_{\mathbf{k}}(y e)_{(1)} n_{(1)} \\
\quad=\sum\left(y_{(0)} e_{(0)} \otimes_{\mathcal{E}} n_{(0)}\right) \otimes_{\mathbf{k}} y_{(1)} e_{(1)} n_{(1)} \\
\quad=\sum\left(y_{(0)} \otimes_{\mathcal{E}}(\text { en })_{(0)}\right) \otimes_{\mathbf{k}} y_{(1)}(e n)_{(1)} \in S^{-1} M \otimes_{\mathbf{k}} \mathcal{B} .
\end{aligned}
$$

Thus we get a functor $Q_{S}^{\mathcal{B}}: \mathcal{E}^{\mathcal{B}} \rightarrow S^{-1} \mathcal{E} \mathcal{M}^{\mathcal{B}}$.

This argument extends to perfect localizations. For more general localizations, when the definition in terms of the ground ring is not appropriate, we say that a localization functor $Q=Q^{*}$ is $\rho$-compatible if it induces a localization functor $Q^{\mathcal{B}}$ on the category of dimodules $\mathcal{E} \mathcal{M}^{\text {co } \mathcal{B}}$. The localization maps $i_{M}: M \rightarrow Q(M)$ then also lift to the maps of dimodules. This may be seen by observing that, abstractly, the localization maps come from the adjunction $i: \operatorname{Id} \rightarrow Q_{*} Q^{*}$.

Apart from easy generalizations, the reformulation in terms of dimodule categories has other useful consequences. For each dimodule $M$, there is an equality $Q_{S}^{\mathcal{B}}\left(Q_{S}^{\mathcal{B}} M\right)=Q_{S}^{\mathcal{B}} M$ of dimodules. A more important observation is that the functors of the type $Q_{S}^{\mathcal{B}}$ can be iterated. This means that there is a natural $\mathcal{B}$-coaction on the successive $\rho$-compatible localizations $S_{1}^{-1} S_{2}^{-1} \cdots S_{n}^{-1} \mathcal{E}$ which is a refinement (cf. discussion of covers by localizations above) of the previous picture where we could do this only for $\left(S_{1} \vee S_{2} \vee \ldots \vee S_{n}\right)^{-1} \mathcal{E}$, as the latter is a ring. We can now define the module of localized coinvariants in $S_{1}^{-1} S_{2}^{-1} \cdots S_{n}^{-1} \mathcal{E}$ as the module of coinvariants for this induced $\mathcal{B}$-coaction.

9. Covers by coaction-compatible localizations. To every cover of $\mathcal{E}$ by flat localizations one associates a cosimplicial object

$$
\mathcal{E} \longrightarrow \prod_{\mu} \mathcal{E}_{\mu} \longrightarrow \prod_{\mu, \nu} \mathcal{E}_{\mu \nu} \longrightarrow \cdots
$$

where $\mathcal{E}_{\mu \nu \ldots}$ are the successive localizations. If the cover is semiseparated then we can identify $\mathcal{E}_{\mu \nu}$ with $\mathcal{E}_{\nu \mu}$ etc. so the products can be taken with $\mu<\nu<\ldots$ with respect to 
any fixed ordering on the indices of the cover. If the localizations are $\rho$-compatible and cover semiseparated this is a cosimplicial object in the category of $\mathcal{B}$-comodule algebras. Without semiseparetedness it is only in the category $\mathcal{E}^{\mathcal{B}}$.

Denote by $\mathcal{E}^{\mathcal{M}} \mathcal{M}_{\mu \nu \ldots}^{\mathcal{B}}$ the category obtained from $\varepsilon_{\mathcal{E}} \mathcal{M}^{\mathcal{B}}$ by successive application of dimodule category localizations $Q_{\mu}^{\mathcal{B}}$. Again, in the semiseparated case, $\mathcal{E}^{\mathcal{M}} \mathcal{M}_{\mu \nu \ldots}$ will agree with the dimodule category $\mathcal{E}_{\mu \nu \ldots} \mathcal{M}^{\mathcal{B}}$, but in general $\mathcal{E}_{\mu \nu \ldots}$ is not a ring, but only an $\mathcal{E}$-bimodule, so the latter category does not make sense. For any flat localization functor $Q^{*}$, its right adjoint $Q_{*}$ is fully faithful [20]. Thus we may view the objects in $\mathcal{E}_{\mathcal{M}} \mathcal{M}_{\mu \nu}^{\mathcal{B}}$ as living in $\mathcal{E}_{\mathcal{M}} \mathcal{M}^{\mathcal{B}}$. In particular, every functor $F$ defined on ${ }_{\mathcal{E}} \mathcal{M}^{\mathcal{B}}$ can be naturally evaluated on $\mathcal{E}_{\mu \nu \ldots}^{\mathcal{B}}$.

More generally, if we replace the $\mathcal{B}$-comodule algebra $\mathcal{E}$ in (6) by any dimodule $M \in$ $\mathcal{E}^{\mathcal{B}}$ we also obtain a cosimplicial object. Moreover, the construction is functorial in $M$. Hence we obtain a cosimplicial object in the category of endofunctors End $\mathcal{E}^{\mathcal{B}}$.

For details on this "standard resolution" construction see [69], remembering that now our objects live in the Abelian (actually Grothendieck [94]) category $\mathcal{E}^{\mathcal{B}}$.

Consider the left exact functor

$$
()^{\operatorname{co} \mathcal{B}}: \mathcal{E}^{\mathcal{B}} \mathcal{M}^{\mathcal{B}} \rightarrow \mathcal{M}
$$

of taking the submodule of coinvariants, where $U=\mathcal{E}^{\text {co } \mathcal{B}}$. The functor $M \mapsto \mathcal{E} \otimes \mathcal{U} M$ is a left adjoint to ()$^{\mathrm{co} \mathcal{B}}$. Here the $\mathcal{E}$-action on $\mathcal{E} \otimes \mathcal{U} M$ extends the map $e\left(e^{\prime} \otimes \mathcal{U} m\right)=e e^{\prime} \otimes \mathcal{U} m$ and the $\mathcal{B}$-coaction extends the map $e \otimes m \mapsto \sum\left(e_{(0)} \otimes_{\mathcal{U}} v\right) \otimes_{\mathbf{k}} e_{(1)}$. The adjunctions are given by

$$
\begin{array}{ll}
M \mapsto\left(\mathcal{E} \otimes_{\mathcal{U}} M\right)^{\mathrm{co} \mathcal{B}}, & m \mapsto 1 \otimes m, \\
\mathcal{E} \otimes_{\mathcal{U}} M^{\mathrm{co} \mathcal{B}} \rightarrow M, & e \otimes_{\mathcal{U}} m \mapsto e m .
\end{array}
$$

H.-J. Schneider's theorem $[75,60]$ says that these two adjoint functors are equivalences iff $\mathcal{E}$ is a faithfully flat Hopf-Galois extension of $\mathcal{U}$. We'll now sketch the interplay between the functors playing a role in the Schneider theorem and the (co)simplicial structures coming from the cover by the coaction-compatible localizations.

Throughout this section we denote $\mathcal{U}^{\mu \nu \ldots}=\left(\mathcal{E}_{\mu \nu \ldots}\right)^{\mathrm{co} \mathcal{B}}$. We use the upper indices, as $\mathcal{U}^{\mu \nu \ldots}$ is not necessarily a localization (lower indices!) of $\mathcal{U}$. In section 8, we have discussed that any $\rho$-compatible localization functor $Q_{\mu}$ induces the localization functor $Q_{\mu}^{\mathcal{B}}$ on the category of dimodules, hence if the functors from the Schneider theorem are equivalences then there is a well defined localized category $\mathcal{u} \mathcal{M}_{\mu \nu \ldots}$ of $\mathcal{u} \mathcal{M}$, but in general $\mathcal{U M}$ is not defined. When such localized categories are defined, we can restate the very fact by saying that the $\rho$-compatible cover $\mathfrak{U}=\left\{Q_{\mu}\right\}$ of $\mathcal{E}$ induces a cover $\tilde{\mathfrak{U}}$ of $\mathcal{U}$. The category of $\mathcal{U}^{\mu \nu \ldots}$-modules, denoted by $\mathcal{U}^{\mu \nu \ldots \mathcal{M}}$ is not necessarily a localized category $\mathcal{U M}_{\mu \nu \ldots}$ of $\mathcal{u} \mathcal{M}$, even when the latter exists (when it exists it is typically bigger than the latter). In other words, taking the coinvariants and localizing do not commute.

A cosimplicial object in $\mathcal{E}_{\mathcal{B}} \mathcal{M}^{\mathcal{B}}$ is given by a functor from the cosimplicial category $\Delta$ to $\mathcal{E}^{\mathcal{B}}$. In our case, to each function $f:[l] \rightarrow[k]$ in the cosimplicial category $\Delta$ one assigns a certain composition $Q_{f}^{\mathcal{B}}$ of the localization functors $Q_{\mu}^{\mathcal{B}}$ (roughly speaking: formulas also involve their right adjoints, the inclusion into the direct product, and the unit of an appropriate adjunction, cf. [47, 69, 70, 93]). 
For each cover $\mathfrak{U}=\left\{Q_{\mu}\right\}$ of $\mathcal{E}$ by $\rho$-compatible Gabriel localizations we define a category $\varepsilon_{\mathcal{M}} \mathcal{M}(\mathfrak{U})^{\mathrm{co} \mathcal{B}}$, the $\mathfrak{U}$-accessible quotient, as follows. For mere sake of comparison, we'll also define two other categories in a similar way, $\mathcal{E}^{\mathcal{B}}(\mathfrak{U}), \mathcal{U} \mathcal{M}(\tilde{\mathfrak{U}})$. Instead of an object $M$ and its successive localizations $M_{\mu \nu \ldots}$ we consider a family $N^{\cdot}$ of objects $N^{\mu \nu \cdots}$ (upper indices!) in categories $\mathcal{C}^{\mu \nu \cdots}$ respectively, given with a family of structure morphisms b. . Here $\mathcal{C}^{\mu \nu \ldots}=\mathcal{U}^{\mu \nu \ldots \mathcal{M}}$ for the $\mathfrak{U}$-accessible quotient $\mathcal{E} \mathcal{M}(\mathfrak{U})^{\operatorname{co} \mathcal{B}}$, and $\mathcal{C}^{\mu \nu \ldots}=$ ${ }_{\mathcal{E}} \mathcal{M}_{\mu \nu \ldots}^{\mathcal{B}}$ and $\mathcal{U} \mathcal{M}_{\mu \nu \ldots}$ for the construction of ${ }_{\mathcal{E}} \mathcal{M}^{\mathcal{B}}(\mathfrak{U})$ and $\mathcal{U} \mathcal{M}(\tilde{\mathfrak{U}})$ respectively. Here $\mathcal{U} \mathcal{M}(\tilde{\mathfrak{U}})$ is defined only when $\tilde{\mathfrak{U}}$ is defined. Denote $N_{k}=\prod_{\mu_{1}, \ldots, \mu_{k}} N^{\mu_{1} \ldots \mu_{k}}$ and $\mathcal{C}_{k}$ the corresponding category. A family of structure morphisms is an assignment $b:(f, k) \mapsto$ $b(f, k)$, where $f:[l] \rightarrow[k]$ is in the cosimplicial category $\Delta, k$ is a natural number and $b(f, k)$ is a morphism in $\mathcal{C}_{k}$,

$$
b(f, k): Q_{f}^{\mathcal{B}}\left(N_{k}\right) \rightarrow N_{l}, \text { such that }\left\{\begin{array}{l}
b(f \circ g, k)=b(g, l) \circ Q_{g}^{\mathcal{B}}(b(f, k)) \\
Q_{f \circ g}^{\mathcal{B}}\left(N_{k}\right)=Q_{g}^{\mathcal{B}} Q_{f}^{\mathcal{B}}\left(N_{k}\right) \rightarrow Q_{g}^{\mathcal{B}}\left(N_{l}\right) \rightarrow N_{m} \\
b(\mathrm{id}, k)=\mathrm{id}
\end{array}\right.
$$

Objects of the category ${ }_{\mathcal{E}} \mathcal{M}(\mathfrak{U})^{\mathrm{co} \mathcal{B}}$ are pairs $\left(N_{\bullet}, b\right)$, as described above, and similarly for the other two categories. A morphism $f_{\bullet}:\left(N_{\bullet}, b\right) \rightarrow\left(N_{\bullet}^{\prime}, b^{\prime}\right)$ is a sequence $\left(f_{k}\right)$, where each $f_{k}: N_{k} \rightarrow N_{k}^{\prime}$ is an $\mathcal{E}_{k}$-module map, and where $f_{k}$ commute with structure morphisms. Composition of morphisms is defined componentwise. This construction is an analogue of the category of simplicial sheaves over a simplicial space, cf. [6, 13, 33, 54].

The functor $M \mapsto\left(N_{\bullet}, b\right)$ defined by $N^{\mu \nu \ldots}=\left(M_{\mu \nu \ldots}\right)^{\operatorname{co} \mathcal{B}}$ and $b=$ id defines a functor ${ }_{\mathcal{E}} \mathcal{M}^{\mathcal{B}} \rightarrow{ }_{\mathcal{E}} \mathcal{M}(\mathfrak{U})^{\mathrm{co} \mathcal{B}}$. The functor $M \mapsto\left(N_{\bullet}, b\right)$ defined by $N^{\mu \nu \ldots}=M_{\mu \nu \ldots}$ from $\mathcal{E}^{\mathcal{B}}$ to $\mathcal{E} \mathcal{M}^{\mathcal{B}}(\mathfrak{U})$ is, however, an equivalence of categories (and all the structure morphisms are isomorphisms). This follows from the globalization lemma (section 5) and general nonsense about simplicial objects (cf. [70], Prop. 1.0.8.2, for the appropriate statement in the language of comonads).

It is a basic phenomenon that taking coinvariants does not commute with localization. However, if ()$^{\operatorname{co} \mathcal{B}}$ is an equivalence of categories, then all the localization functors $Q_{\mu}^{\mathcal{B}}$ may be viewed as localization functors in the category $\mathcal{u} \mathcal{M}$. By the globalization lemma (now in $\mathcal{U M}), \mathcal{u} \mathcal{M}=\mathcal{U} \mathcal{M}(\tilde{\mathfrak{U}})$, where $\tilde{\mathfrak{U}}$ is the localization cover induced by $\mathfrak{U}$ as above. Hence ${ }_{\mathcal{E}} \mathcal{M}^{\mathcal{B}}$ is equivalent to $\mathcal{E}_{\mathcal{M}}(\mathfrak{U})^{\operatorname{co} \mathcal{B}}$. If ()$^{\operatorname{co} \mathcal{B}}$ is not an equivalence, but ()$^{\mathrm{co} \mathcal{B}}$ of each of the categories $\mathcal{E}_{\mathcal{M}}^{\mathcal{B}}$ is (each $\mathcal{E}_{\mu}$ is a faithfully flat Hopf-Galois extension), then $\mathcal{E}^{\mathcal{B}}$ is still equivalent to $\mathcal{E} \mathcal{M}(\mathfrak{U})^{\text {co } \mathcal{B}}$.

One thinks of the category of Hopf modules ${ }_{\mathcal{E}} \mathcal{M}^{\mathcal{B}}$ as the category $Q \operatorname{coh}_{B}(E)$ of $B$ equivariant (for right $B$-action, where $B$ is an affine group and $\mathcal{B}=\mathcal{O}(B)$ ) quasicoherent sheaves on an affine $B$-variety $E$. If the action is free and under some flatness conditions, classical descent theory [28] identifies $Q \operatorname{coh}_{B}(E)$ with the category $Q \operatorname{coh}(E / B)$ (in that case, "descent is effective") of quasicoherent sheaves on the quotient space. The latter category is approximated by the $\mathfrak{U}$-accessible quotient, and achieved in the case of Schneider's equivalence in each localization of the cover. In a sense, this is a globalization of Schneider's equivalence, i.e. a special case of the (effective) descent method for a noncommutative nonaffine base (quotient) space.

One could naively always define $Q \operatorname{coh}(E / B)$ as being simply ${ }_{\mathcal{E}} \mathcal{M}^{\mathcal{B}}$. However, this may not be always achieved, if we want to equip this category with local coordinate charts. 
A comodule algebra $\mathcal{E}$ itself (put coaction aside for a moment) does not only define the category of $\mathcal{E}$-modules, it amounts also to a choice of a Morita equivalence class of the underlying ring of that category. A global version of that choice is achieved as follows.

$\mathcal{E}$ is viewed as a relative noncommutative scheme [69] over the category of modules over $\mathbf{k}$. The inverse image of the distinguished object in the category of $\mathbf{k}$-modules is the structure sheaf $\mathcal{O}_{\mathcal{E}}$. For any perfect localization $Q$, the inverse image $Q^{*}\left(\mathcal{O}_{\mathcal{E}}\right)$ is a $\mathbf{k}$-algebra. One wants a noncommutative quotient scheme $E / B$ over $\mathbf{k}$ also equipped with $\mathcal{O}_{E / B}$. Realizing this structure sheaf for some category close to $\mathcal{E}^{\mathcal{B}}$ is the basic meaning of the construction of the quotient space. Our approach is to localize enough to get $\mathcal{O}_{E / B}$ from local coinvariants, or at least to approximate it. "Size" of an $\mathfrak{U}$-accessible quotient is a naive measure of the approximation. An ideal case is when each localization of $\mathcal{E}$ is a Hopf-Galois extension: intuitively, the local coinvariants then patch to the full structure sheaf. One can extend the theory beyond the affine case. A (nonaffine) noncommutative $\mathbf{k}$-scheme $\operatorname{Spec}(\mathcal{E})$ is required to come with an affine cover by compatible perfect localizations. Those are given coactions (on the pieces of $\mathcal{O}_{\mathcal{E}}$ ). The coaction should agree when viewed at the level of categories of modules (in the commutative case only some $B$-schemes are of this form, but it is strictly larger than affine $B$-schemes). To achieve further progress it is promising to rely on simplicial and cohomological methods; the ideas of cohomological descent [13] and the noncommutative Cech cohomologies [69, 92] provide a framework. Cf. also $[3,6,12,25,33,45,54,55,55]$. Very recently, V. Lunts suggested that it might be useful to consider more general flat covers and resolutions of would-be quotient space than the covers coming from localizations, and to use the corresponding (co)simplicial objects to define and investigate quotients of comodule algebras in full generality [46] (even nontrivial $\rho$-compatible localization covers are not always available). However, in the rest of the paper, I will present my earlier work focusing on the localization approach, which for some purposes suffices.

10. Quantum bundles. A right (left) Zariski locally trivial principal $\mathcal{B}$-bundle is a right (left) $\mathcal{B}$-comodule algebra $(\mathcal{E}, \rho)$ together with a right (left) Zariski local trivialization. A local right (left) trivialization of $(\mathcal{E}, \rho)$ consists of

- a cover of $\mathcal{E}$ by $\rho$-compatible perfect localizations $i_{\lambda}: \mathcal{E} \rightarrow \mathcal{E}_{\lambda}$,

- a family $\left\{\gamma_{\lambda}: \mathcal{B} \rightarrow \mathcal{E}_{\lambda}\right\}$ of right (left) $\mathcal{B}$-comodule algebra homomorphisms.

Here the $\mathcal{B}$-comodule structure on $\mathcal{E}_{\lambda}$ is the one induced by $\rho$-compatibility.

Local triviality implies that each $\left(\mathcal{E}_{\lambda}, i_{\lambda}, \gamma_{\lambda}\right)$ is in the category of higher smash products $\mathcal{C}(U, \mathcal{B})$ for some $\mathcal{U}$ (trivial quantum principal bundle).

Let now $\left(M, \rho_{M}\right)$ be a left $\mathcal{B}$-comodule. Define

$$
\begin{aligned}
& \kappa_{\lambda}=(\mu \otimes \mathrm{id})\left(\mathrm{id} \otimes \gamma_{\lambda} \otimes \mathrm{id}\right)\left(\mathrm{id} \otimes \rho_{M}\right): \mathcal{E}_{\lambda}^{\mathrm{co} \mathcal{B}} \otimes M \rightarrow \mathcal{E}_{\lambda} \otimes M, \\
& \bar{\kappa}_{\lambda}=(\mu \otimes \mathrm{id})\left(\mathrm{id} \otimes\left(\gamma_{\lambda} \circ S\right) \otimes \mathrm{id}\right)\left(\mathrm{id} \otimes \rho_{M}\right): \mathcal{E}_{\lambda} \otimes M \rightarrow \mathcal{E}_{\lambda} \otimes M .
\end{aligned}
$$

Equivalently, $\kappa_{\lambda}$ is the unique map such that

$$
\kappa_{\lambda}(f \otimes m)=\sum f \gamma_{\lambda}\left(m_{(-1)}\right) \otimes m_{(0)} .
$$

These data are enough to define the associated (vector) bundle $\xi_{M}$, when the cover $\{\lambda \in \Lambda\}$ is finite. Denote $\Gamma_{\lambda} \xi_{M}=\mathcal{E}_{\lambda}^{\text {co } \mathcal{B}} \otimes M$ and define $\Gamma_{\lambda} \xi_{M}$ to be a $\mathbf{k}$-space of local 
sections of associated bundle over localization $\lambda$. For other localizations $Q_{\mu}$ (not included in the trivializing cover), a version of the globalization lemma (Theorem 6.2 in [71]) and elementary arguments justify the definition by a " $\kappa$-twisted descent" formula

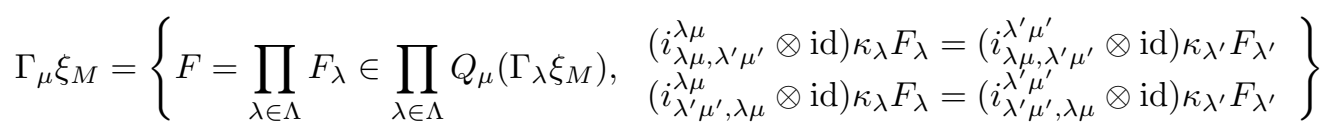

This said, below we limit ourselves to the original cover and the global sections.

Proposition 1. $\bar{\kappa}_{\lambda} \circ \kappa_{\lambda}=\mathrm{id}$. In particular, $\kappa_{\lambda}$ is an isomorphism onto its image and $\left.\kappa_{\lambda} \circ \bar{\kappa}_{\lambda}\right|_{\operatorname{Im} \kappa_{\lambda}}=\mathrm{id}$.

The cotensor product of a left $\mathcal{B}$-comodule $\mathrm{M}$ and right $\mathcal{B}$-comodule $\mathcal{E}$ is defined by

$$
\mathcal{E} \square_{\mathcal{B}} M=\left\{H \in \mathcal{E} \otimes M \mid\left(\mathrm{id} \otimes \rho_{M}\right) H=\left(\rho_{E} \otimes \mathrm{id}\right) H\right\} .
$$

The cotensor product has a priori only a structure of a $\mathbf{k}$-vector space. If $\mathcal{E}$ is a $\mathcal{B}$-comodule algebra which is the total space of a Hopf-algebraic trivial principal bundle (which we identify with the smash product $\mathcal{E}^{\mathrm{co} \mathcal{B}} \sharp \mathcal{B}$ ), then it is elementary that $\mathcal{E}^{\mathrm{co} \mathcal{B}} \otimes M$ is canonically isomorphic to the cotensor product as a $\mathbf{k}$-vector space. We extend this result to the setting of locally trivial bundles.

Lemma $2[76,77]$. The image of $\mathcal{E} \square_{\mathcal{B}} M$ in the localization $\mathcal{E}_{\lambda} \otimes M$ is contained in $\operatorname{Im} \kappa_{\lambda}$.

The algebra of global sections of the associated bundle $\Gamma \xi_{M}$ is (in this setup) given by

$$
\Gamma \xi_{M}=\left\{F=\prod_{\lambda \in \Lambda} F_{\lambda} \in \prod_{\lambda \in \Lambda} \Gamma_{\lambda} \xi_{M}, \begin{array}{l}
\left(i_{\lambda, \lambda^{\prime}}^{\lambda} \otimes \mathrm{id}\right) \kappa_{\lambda} F_{\lambda}=\left(i_{\lambda, \lambda^{\prime}}^{\lambda^{\prime}} \otimes \mathrm{id}\right) \kappa_{\lambda^{\prime}} F_{\lambda^{\prime}} \\
\left(i_{\lambda^{\prime}, \lambda}^{\lambda} \otimes \mathrm{id}\right) \kappa_{\lambda} F_{\lambda}=\left(i_{\lambda^{\prime}, \lambda}^{\lambda^{\prime}} \otimes \mathrm{id}\right) \kappa_{\lambda^{\prime}} F_{\lambda^{\prime}}
\end{array}\right\} .
$$

TheOREM 3. $\Gamma \xi_{M}$ is naturally isomorphic to $\mathcal{E} \square_{\mathcal{B}} M$ as a $\mathbf{k}$-vector space.

Proof. By the globalization lemma, there is an isomorphism $i_{\Lambda}$ from $\mathcal{E}$ to the equalizer of the diagram $\prod \mathcal{E}_{\lambda} \rightrightarrows \prod \mathcal{E}_{\lambda, \lambda^{\prime}}$. Tensoring $i_{\Lambda}$ and the equalizer diagram with id ${ }_{M}$ over the ground field $\mathbf{k}$ yields an isomorphism $i_{\Lambda} \otimes \operatorname{id}_{M}$ from $\mathcal{E} \otimes M$ to the equalizer of $\prod \mathcal{E}_{\lambda} \otimes M \rightrightarrows \prod \mathcal{E}_{\lambda, \lambda^{\prime}} \otimes M$. A direct check shows that $\prod \rho_{\mathcal{E}_{\lambda}} \otimes \mathrm{id}_{M}$ and $\prod \mathrm{id}_{\mathcal{E}_{\lambda}} \otimes \rho_{M}$ agree on the image of map $\bar{\kappa}_{\lambda}\left(i_{\lambda} \otimes \operatorname{id}_{M}\right)$ defined on $\prod_{\lambda} \mathcal{E}_{\lambda}^{\text {co } \mathcal{B}} \otimes M$. As our localization endofunctors are flat, and the coactions $\rho_{\mathcal{E}_{\lambda}}$ extend $\rho_{\mathcal{E}}$, the covering property implies that $\prod \rho_{\mathcal{E}} \otimes \operatorname{id}_{M}$ agrees with $\prod \operatorname{id}_{\mathcal{E}_{\lambda}} \otimes \rho_{M}$ in the equalizer. The isomorphism $\Gamma \xi_{M} \rightarrow \mathcal{E} \square_{\mathcal{B}} M$ is well defined by $\left(i_{\Lambda} \otimes \operatorname{id}_{M}\right)^{-1} \prod_{\lambda} \kappa_{\lambda}$. Namely, its inverse is $H \mapsto \prod_{\lambda} \bar{\kappa}_{\lambda}\left(i_{\lambda} \otimes \operatorname{id}_{M}\right) H$. This follows by Lemma 2 and the properties of $\kappa_{\lambda}$-s listed above.

Corollary 4. Let $\mathcal{G}$ be an Hopf algebra, $\mathcal{B}$ a quantum subgroup (quotient Hopf algebra). If $\left(\mathcal{G}_{\lambda}, \rho_{\mathcal{B}}\right)$ is a Zariski locally trivial principal $\rho_{\mathcal{B}}$-bundle, then the inducing functor for comodules $\operatorname{Ind}_{\mathcal{B}}^{\mathcal{G}}: \mathcal{M}^{\mathcal{B}} \rightarrow \mathcal{M}^{\mathcal{G}}$ can be realized by gluing local sections.

Here $\pi: \mathcal{G} \rightarrow \mathcal{B}$ is an onto map of Hopf algebras. The induced comodule can be described as $\operatorname{Ind}_{\mathcal{B}}^{\mathcal{G}} M=\mathcal{G} \square_{\mathcal{B}} M$ where $\rho_{\mathcal{B}}: \mathcal{G} \rightarrow \mathcal{G} \otimes \mathcal{B}$ is given by $\rho_{\mathcal{B}}=(\mathrm{id} \otimes \pi) \Delta_{\mathcal{G}}$.

One can interpret the global sections functor $\Gamma$ as an appropriate 0 -th Cech-type cohomology $\check{H}^{0}$. Similarly, the application of the methods in [69] implies a generalization of Corollary 4: the composition $\check{H}^{i} \circ \xi_{-}$, where $\check{H}^{i}$ are the appropriate higher Cech-type 
cohomologies and $\xi_{-}$is the associated bundle functor $M \mapsto \xi_{M}$, realize the higher derived functors $R^{i} \operatorname{Ind}_{B}^{G}$ of the induction functor for comodules.

We use (co)matrix notation for coaction to introduce the transition matrices for $\xi_{M}$. Supose $\left\{m_{\beta}\right\}$ is a basis of $M$ and the $\mathcal{B}$-coaction is given by

$$
\rho: m_{\beta} \mapsto \sum_{\alpha} m_{\beta}^{\alpha} \otimes m_{\alpha}
$$

Represent $F_{\lambda} \in \Gamma_{\lambda} \xi_{M}$ as

$$
F_{\lambda}=\sum_{\alpha} f_{\lambda}^{\alpha} \otimes m_{\alpha}
$$

The condition $\kappa_{\lambda} F_{\lambda}=\kappa_{\lambda^{\prime}} F_{\lambda^{\prime}}$ (in both consecutive localizations) reads

$$
\sum_{\alpha, \beta} f_{\lambda}^{\alpha} \gamma_{\lambda}\left(m_{\alpha}^{\beta}\right) \otimes m_{\beta}=\sum_{\alpha, \beta} f_{\lambda^{\prime}}^{\alpha} \gamma_{\lambda^{\prime}}\left(m_{\alpha}^{\beta}\right) \otimes m_{\beta} .
$$

As $\left\{m_{\beta}\right\}$ is a basis, this is equivalent to the statement

$$
(\forall \beta) \sum_{\alpha} f_{\lambda}^{\alpha} \gamma_{\lambda}\left(m_{\alpha}^{\beta}\right)=\sum_{\alpha} f_{\lambda^{\prime}}^{\alpha} \gamma_{\lambda^{\prime}}\left(m_{\alpha}^{\beta}\right) .
$$

Multiplying this identity by $\gamma_{\lambda}\left(S m_{\beta}^{\gamma}\right)$ and summing over $\beta$ we obtain

$$
\sum_{\alpha, \beta} f_{\lambda}^{\alpha} \gamma_{\lambda}\left(m_{\alpha}^{\beta}\right) \gamma_{\lambda}\left(S m_{\beta}^{\gamma}\right)=\sum_{\alpha, \beta} f_{\lambda^{\prime}}^{\alpha} \gamma_{\lambda^{\prime}}\left(m_{\alpha}^{\beta}\right) \gamma_{\lambda}\left(S m_{\beta}^{\gamma}\right)
$$

As $\gamma_{\lambda}$ is an algebra map, $\sum_{\alpha} \gamma_{\lambda}\left(m_{\alpha}^{\beta}\right) \gamma_{\lambda}\left(S m_{\beta}^{\gamma}\right)=\delta_{\alpha}^{\gamma} 1$, which simplifies the left-hand side to $f_{\lambda}^{\gamma}$. Denote

$$
\left(\mathfrak{M}_{\lambda^{\prime}, \lambda}\right)_{\alpha}^{\gamma}:=\sum_{\beta} \gamma_{\lambda^{\prime}}\left(m_{\alpha}^{\beta}\right) \gamma_{\lambda}\left(S m_{\beta}^{\gamma}\right) \in \mathcal{E}_{\lambda \vee \lambda^{\prime}}
$$

This defines a matrix $\mathfrak{M}_{\lambda, \lambda^{\prime}}$ which is called the transition matrix between the charts $\lambda$ and $\lambda^{\prime}$. In this notation (8) becomes

$$
f_{\lambda}^{\gamma}=\sum_{\alpha} f_{\lambda^{\prime}}^{\alpha}\left(\mathfrak{M}_{\lambda^{\prime}, \lambda}\right)_{\alpha}^{\gamma}
$$

It follows from definition (9) that

$$
\mathfrak{M}_{\lambda, \lambda}=I, \quad \mathfrak{M}_{\lambda, \mu} \mathfrak{M}_{\mu, \nu}=\mathfrak{M}_{\lambda, \nu}
$$

where the upper index of $\mathfrak{M}_{\lambda^{\prime}, \lambda}$ is the column index rather than the row index (the latter would be along conventions for other matrices in this work).

Finally, we comment on the restriction of (algebras of global sections of) bundles to Ore localizations.

TheOrem $5[76,77]$. There exist a Hopf algebra $\mathcal{B}$ and a $\mathcal{B}$-comodule algebra $(\mathcal{E}, \rho)$ such that

- The algebra of (global) coinvariants $\mathcal{E}^{\mathrm{co} \mathcal{B}}$ is commutative (possible to choose the polynomial ring $\mathbf{k}[u]$ ).

- $\mathcal{E}$ is isomorphic to a smash product of $\mathcal{B}$ and $\mathcal{E}^{\mathrm{co} \mathcal{B}}$ with coaction $\otimes \Delta$.

- There exists a multiplicative set $U \subset \mathcal{E}^{\mathrm{co} \mathcal{B}}$ (automatically Ore by commutativity) such that there is no pair of a $\rho$-compatible left Ore set $S \subset \mathcal{E}$ and an isomorphism 
$\zeta$ of $\mathcal{B}$-comodule algebras

$$
\zeta:\left(S^{-1} \mathcal{E}\right)^{\mathrm{co} \mathcal{B}} \rightarrow U^{-1}\left(\mathcal{E}^{\mathrm{co} \mathcal{B}}\right)
$$

such that $\left.\zeta \circ i_{S}\right|_{\mathcal{E}^{\mathrm{co} \mathcal{B}}}=i_{U}$.

This phenomenon is nonexistent in the commutative case and generic in the noncommutative case, even if, as here, all the global coinvariants pairwise commute. In [58] some conditions preventing such phenomena were considered for certain kinds of localizations using filters of ideals. In [45] graded actions and actions by differential operators were considered and, in their setup, guaranteed that module algebra actions extend to localizations. In the language of modules and dimodules, the simplest reasoning about these phenomena is as follows. The coaction on the whole ring does not necessarily extend to the localized ring as we know. If it does, then the localization induces the functor $Q^{\mathcal{B}}$ on $\mathcal{E}^{\mathcal{B}}$, so if the latter category is equivalent to $\mathcal{u} \mathcal{M}$ (say, in the faithfully flat Hopf-Galois case), we obtain an induced localization in the base for free. The induced radical $\sigma_{U}$ in the base category $\mathcal{U} \mathcal{M}$ is given by $M \mapsto N$ whenever $\sigma^{\mathcal{B}}: M \otimes \mathcal{B} \mapsto N \otimes \mathcal{B}$. One cannot a priori say if the induced localization of $\mathcal{U} \mathcal{M}$ is of some specific type. Say, if we start with a $\rho$-compatible Ore localization of $\mathcal{E}_{\mathcal{M}} \mathcal{M}$, is the induced localization of $\mathcal{U} \mathcal{M}$ Ore or only a perfect localization? This is not a very tractable question. A $\mathcal{B}$-comodule algebra $(\mathcal{E}, \rho)$ is sufficiently localized with respect to the family of all $\rho$-compatible left Ore localizations if for every such $S$ there is a left Ore set $U$ in $\mathcal{U}=\mathcal{E}^{\text {co } \mathcal{B}}$ such that

$$
i_{S} \mid \mathcal{E}^{\mathcal{B}}: \mathcal{E}^{\mathcal{B}} \rightarrow\left(S^{-1} \mathcal{E}\right)^{\mathcal{B}}
$$

is canonically isomorphic to an Ore localization of $\mathcal{E}^{\mathcal{B}}$ with respect to some Ore set $U \subset \mathcal{E}^{\mathcal{B}}$. It is interesting to compare this notion with other mentioned conditions on the relation between the base and total algebra. For example, by the fundamental theorem of Hopf modules, each $\rho$-compatible flat localization of ${ }_{\mathcal{B}} \mathcal{M}^{\mathcal{B}}$ is equivalent to a flat localization of the category of $\mathbf{k}$-vector spaces. As all such localizations are trivial, it is not possible to find a nontrivial Ore localization $\mathcal{G}\left[T^{-1}\right]$ of a Hopf algebra $\mathcal{G}$ such that the comultiplication $\Delta_{\mathcal{G}}$ extends multiplicatively to a coaction $\Delta_{T}$ in the localization. This is an extension of the basic fact that the only $G$-invariant subset of an algebraic group $G$ is $G$ itself.

Start with a localization $Q_{\mathcal{U}}$ of the base category $\mathcal{U M}$, and suppose it is abstractly equivalent to the category of dimodules $\mathcal{E}^{\mathcal{B}}$ on the total algebra $\mathcal{E}$. Then, of course, we have a localization $Q^{\mathcal{B}}$ on that category, but there is no a priori reason to believe that $Q^{\mathcal{B}}$ is induced by any localization $Q$ defined on the ambient category $\mathcal{M}$. For example, the same module $M$ can have two different Hopf module structures $M_{1}$ and $M_{2}$, and $Q^{\mathcal{B}}\left(M_{1}\right)$ may disagree with $Q^{\mathcal{B}}\left(M_{2}\right)$ as an $\mathcal{E}$-module. If the abstract category equivalence is however the natural one - as in the Schneider theorem, that is, via ()$^{\text {co } \mathcal{B}}$ and $\otimes_{\mathbf{k}} \mathcal{B}$ - then this bad phenomenon is prevented.

Ore sets in the coinvariants algebra $\mathcal{U}$ (for a trivial bundle) may correspond to more general (than Ore) $\rho$-compatible localizations in the total space, so taking just the "topology" generated by Ore localizations would mean that the geometric projection is not continuous in the obvious sense, even in otherwise well-behaved noncommutative examples. 
11. Cohn localization $[9,10]$. Let $R$ be a possibly noncommutative ring, and $\Sigma$ a given set of square matrices of possibly different sizes with entries in $R$. A map $f: R \rightarrow S$ of rings is $\Sigma$-inverting if each matrix in $\Sigma$ is mapped to an invertible matrix over $S$. A $\Sigma$-inverting ring map $i_{\Sigma}: R \rightarrow R_{\Sigma}$ is called Cohn localization (or universal $\Sigma$-inverting localization) if for every $\Sigma$-inverting ring map $f: R \rightarrow S$ there exists a unique ring map $\tilde{f}: R_{\Sigma} \rightarrow S$ such that $f=\tilde{f} \circ i_{\Sigma}$.

A set $\Sigma$ of matrices is called multiplicative if $1 \in \Sigma$ and, for any $A, B \in \Sigma$ and a matrix $C$ of right size over $R$, the matrix $\left(\begin{array}{cc}A & C \\ 0 & B\end{array}\right) \in \Sigma$. If $\Sigma$ is multiplicative, then the elements of $R_{\Sigma}$ can be obtained as the components of the solutions to all the matrix equations $A u=a$, where $a$ is a column over $R$ and $A \in \Sigma$. To any multiplicative $\Sigma$, P. M. Cohn [10] associates an idempotent preradical $\sigma_{\Sigma}$. Its left-module variant is given by

$$
M \mapsto \sigma_{\Sigma}(M)=\left\{m \in M \mid \exists u=\left(u_{1}, \ldots, u_{n}\right)^{T}, \exists i, m=u_{i} \text { and } \exists A \in \Sigma, A u=0\right\} .
$$

The associated torsion theory is not always hereditary (i.e. a submodule of a $\sigma_{\Sigma}$-torsion module is not necessarily $\sigma_{\Sigma}$-torsion). Equivalently, $\sigma_{\Sigma}$ is not necessarily left exact. Hereditary torsion theories correspond to Gabriel localizations. In practice, the main problem with Cohn localization is that it is usually hard to determine the kernel of the localization map.

12. Quasideterminants and noncommutative Gauss decomposition. Inverting matrices with noncommutative entries plays a role not only in Cohn localization but also generally in solving linear systems of equations in noncommutative variables. The main tool to do calculations with such matrix inverses and perform Gauss-type decompositions are quasideterminants of Gel'fand and Retakh [22, 41, 23, 24, 76].

Let $A \in M_{n}(R)$ be an $n \times n$ matrix over an arbitrary noncommutative unital associative ring $R$. Suppose the rows and columns of $A$ are labeled. Let us choose a row label $i$ and a column label $j$. By $A_{\hat{j}}^{\hat{i}}$ we'll denote the $(n-1) \times(n-1)$ matrix obtained from $A$ by removing the $i$-th row and the $j$-th column. The $(i, j)$-th quasideterminant $|A|_{i j}$ is

$$
|A|_{i j}=a_{j}^{i}-\sum_{k \neq i, l \neq j} a_{l}^{i}\left(A_{\hat{j}}^{\hat{i}}\right)_{l k}^{-1} a_{j}^{k}
$$

provided the right-hand side is defined.

At most $n^{2}$ quasideterminants of a given $A \in M_{n}(R)$ may be defined. If all the $n^{2}$ quasideterminants $|A|_{i j}$ exist and are invertible then the inverse $A^{-1}$ of $A$ exists in $A \in M_{n}(R)$ and

$$
\left(|A|_{j i}\right)^{-1}=\left(A^{-1}\right)_{j}^{i}
$$

Suppose now we are given an equation of the form

$$
A x=\xi
$$

Define $A(j, \xi)$ as the $n \times n$ matrix whose entries are the same as of $A$ except that the $j$-th column is replaced by $\xi$. Then the noncommutative left Cramer rule says

$$
|A|_{i j} x^{j}=|A(j, \xi)|_{i j}
$$


Quasideterminants for classical matrices are up to sign ratios of the determinant of a matrix and the determinant of an $(n-1) \times(n-1)$ submatrix, as one can see by remembering the formula for matrix inverse in terms of cofactor matrices.

Quasideterminants satisfy a number of useful identities, namely, analogues of classical Muir's law of extensionality, Jacobi inversion formula, Laplace expansion formulas etc. There is also a new property called heredity. Descriptively it is a compatibility of taking quasideterminant with partitioning a matrix (into square matrix blocks). In other words, one can take quasideterminants of partitioned matrices in stages.

Noncommutative Gauss decomposition of a matrix $G$ is the decomposition

$$
G=U A
$$

where $A$ is a lower triangular matrix (with possibly noncommutative entries) and $U$ is an upper unidiagonal matrix (i.e. $u_{j}^{i}=0$ for $i>j$, and $u_{j}^{i}=1$ for $i=j$ ).

The problem (11) is equivalent to the set of $n^{2}$ equations

$$
g_{j}^{i}=\left\{\begin{array}{l}
a_{j}^{i}+\sum_{k>i \geq j} u_{k}^{i} a_{j}^{k}, \quad i \geq j \\
\sum_{k \geq j>i} u_{k}^{i} a_{j}^{k}, \quad i<j .
\end{array}\right.
$$

In terms of quasideterminants the solution is

$$
\begin{aligned}
& a_{j}^{i}=\left|\begin{array}{cccc}
g_{j}^{i} & g_{i+1}^{i} & \cdots & g_{n}^{i} \\
g_{j}^{i+1} & g_{i+1}^{i+1} & \cdots & g_{n}^{i+1} \\
\cdots & \cdots & \cdots & \cdots \\
g_{j}^{n} & g_{i+1}^{n} & \cdots & g_{n}^{n}
\end{array}\right|_{i j}, \quad i \geq j \\
& u_{j}^{i}=\left|\begin{array}{cccc}
g_{j}^{i} & g_{j+1}^{i} & \cdots & g_{n}^{i} \\
g_{j}^{j+1} & g_{j+1}^{j+1} & \cdots & g_{n}^{j+1} \\
\cdots & \cdots & \cdots & \cdots \\
g_{j}^{n} & g_{j+1}^{n} & \cdots & g_{n}^{n}
\end{array}\right|_{i j}\left|\begin{array}{cccc}
g_{j}^{j} & g_{j+1}^{j} & \cdots & g_{n}^{j} \\
g_{j}^{j+1} & g_{j+1}^{j+1} & \cdots & g_{n}^{j+1} \\
\cdots & \cdots & \cdots & \ldots \\
g_{j}^{n} & g_{j+1}^{n} & \cdots & g_{n}^{n}
\end{array}\right|_{j j} \quad i<j,
\end{aligned}
$$

whenever all the principal (= lower right corner) quasiminors (= quasideterminants of submatrices) exist and they are invertible. We suggest to the reader to picture (positions of) rows and columns in $G$ which are involved in the submatrices of $G$ in (12).

\section{Matrix bialgebras and Hopf algebras}

13.1. The full ring $M(n, \mathbf{k})$ of $n \times n$ matrices with (commutative) entries in a field $\mathbf{k}$ is isomorphic to $\mathbf{k}^{n^{2}}$ as a $\mathbf{k}$-vector space. This isomorphism induces a structure of affine $\mathbf{k}$-variety on $M(n, \mathbf{k})$. The regular functions on that variety are polynomials in matrix entries. Introduce $n^{2}$ regular functions

$$
t_{j}^{i}: M(n, \mathbf{k}) \rightarrow \mathbf{k}, \quad t_{j}^{i}(a)=a_{j}^{i}, \quad a \in M(n, \mathbf{k}), \quad i, j=1, \ldots, n .
$$

Then $\mathcal{O}(M(n, \mathbf{k})) \cong \mathbf{k}\left[t_{1}^{1}, t_{2}^{1}, \ldots, t_{n}^{n}\right]$ is the ring of global regular functions on $M(n, \mathbf{k})$.

Let $\mathcal{G}$ be a bialgebra, possibly noncommutative, over a field $\mathbf{k}$ and $G=\left(g_{j}^{i}\right)_{j=1, \ldots, n}^{i=1, \ldots, n}$ an $n \times n$-matrix over $\mathcal{G}$. $\mathcal{G}$ is a matrix bialgebra with basis $G$ if the set of entries of $G$ generates $\mathcal{G}$ and if the comultiplication $\Delta$ and counit $\epsilon$ satisfy

$$
\begin{array}{lll}
\Delta G=G \otimes G, & \text { i.e. } & \Delta g_{j}^{i}=\sum_{k=1}^{n} g_{k}^{i} \otimes g_{j}^{k}, \\
\epsilon G=\mathbf{1}, & \text { i.e. } & \epsilon\left(g_{j}^{i}\right)=\delta_{j}^{i} .
\end{array}
$$


The free (noncommutative) associative algebra $F$ on $n^{2}$ generators $f_{j}^{i}$ has a unique coalgebra structure making it a matrix bialgebra with basis $\left(f_{j}^{i}\right)_{j=1, \ldots, n}^{i=1, \ldots, n}$. We call it the free matrix bialgebra of rank $n^{2}$. Every bialgebra quotient of that bialgebra is a matrix bialgebra.

13.2. Free Hopf algebras. We are going to sketch the construction of free Hopf algebras due to Takeuchi [87]. Let $C$ be a coalgebra over $\mathbf{k}$. Let $C_{i}=C$ for $i$ an even nonnegative integer, and $C_{i}=C_{i}^{\text {cop }}$ (the cooposite coalgebra of $C$ ) for an odd positive integer. Then define $V$ to be the external direct sum (coproduct) of the coalgebras $C_{i}$,

$$
V=\coprod C_{i}
$$

The tensor algebra $T(V)$ of $V$, as of any other coalgebra, has a unique bialgebra structure such that the natural inclusion $i_{V}: V \rightarrow T(V)$ is a coalgebra map. We have $T\left(V^{\text {cop }}\right) \cong$ $T(V)^{\text {cop }}$. Then define a k-linear map

$$
S_{V}: V \rightarrow V^{\text {cop }} \quad \text { by }\left(v_{0}, v_{1}, v_{2}, \ldots\right)=\left(0, v_{0}, v_{1}, v_{2}, \ldots\right) .
$$

There is a unique bialgebra map $S: T(V) \rightarrow T(V)^{\text {cop }}$ extending $S_{V}$. Let $I_{S}$ be the 2-sided ideal in $T(V)$ generated by all elements of the form

$$
\sum c_{(1)} S\left(c_{(2)}\right)-\epsilon(c) 1 \text { and } \sum S\left(c_{(1)}\right) c_{(2)}-\epsilon(c) 1, c \in C_{i}, i=1,2, \ldots
$$

This 2-sided ideal is a biideal and $S\left(I_{S}\right) \subset I_{S}$, hence it induces a bialgebra map

$$
S: T(V) / I_{S} \rightarrow\left(T(V) / I_{S}\right)^{\text {cop }} .
$$

It follows that $H(C)=T(V) / I_{S}$ is a Hopf algebra, the free Hopf algebra on $C$. For any Hopf algebra $H^{\prime}$ and a coalgebra map $\phi: C \rightarrow H^{\prime}$ there is a unique Hopf algebra map $\phi^{\prime}: H(C) \rightarrow H^{\prime}$ such that $\phi^{\prime} \circ i=\phi$ where $i: C \rightarrow H(C)$ is the composition of the inclusion into $T(V)$ and the projection $T(V) \rightarrow H(C)$.

Notice that, for any family $\left\{C_{i}\right\}$ of coalgebras, $T\left(\coprod_{i} C_{i}\right)=\coprod_{i} T\left(C_{i}\right)$ where the coproduct on the RHS is the coproduct in the category of bialgebras, which is the free product of algebras with the natural induced coalgebra structure. In the case when the index set is the nonnegative integers and $C_{i+1}=C_{i}^{\mathrm{cop}}$, the LHS specializes to an intermediate stage in building $H(C)$, so it is a generalization of it.

Manin [50] generalized the RHS. He replaces $T\left(C_{i}\right)$ by any bialgebra $B_{i}$ with $B_{i+1}=$ $B_{i}^{\text {cop,op }}$. Notice that the algebra structure is also opposite between even and odd cases (superfluous/unvisible condition in the case of $T\left(C_{i}\right)$ ). Let $\mathbf{B}=\coprod_{i} B_{i}$ and $S: \mathbf{B} \rightarrow \mathbf{B}$ be again defined by shift in index by +1 . Then the 2 -sided ideal $I_{S} \subset \mathbf{B}$ generated by

$$
\sum b_{(1)} S\left(b_{(2)}\right)-\epsilon(b) 1 \text { and } \sum S\left(b_{(1)}\right) b_{(2)}-\epsilon(b) 1, b \in B_{i}
$$

is $S$-stable and $H(B)=\mathbf{B} / I_{S}$ is a Hopf algebra, the Hopf envelope of $B$.

It satisfies the following universal property: for any Hopf algebra $H^{\prime}$ and a bialgebra map $\phi: B_{0} \rightarrow H^{\prime}$ there is a unique Hopf algebra map $H(\phi): H(B) \rightarrow H^{\prime}$ such that $H(\phi) \circ i=\phi$ where $i: B_{0} \rightarrow H(B)$ is the composition of the inclusion into $\mathbf{B}$ and the projection $\mathbf{B} \rightarrow H(B)$.

13.3. Matrix Hopf algebras. One can specialize the construction of Hopf envelope to any matrix bialgebra on a basis $T=\left(t_{j}^{i}\right)_{j=1, \ldots, n}^{i=1, \ldots, n}$. A matrix Hopf algebra $\mathcal{G}$ with basis 
$T=\left(t_{j}^{i}\right)$ is a Hopf algebra which has a matrix subbialgebra $B$ with basis $T$ such that the map $H(\mathrm{id}): H(B) \rightarrow \mathcal{G}$ is onto. $B$ is not necessarily a matrix bialgebra with respect to that basis: e.g. the commutative coordinate ring of $G L(n, \mathbf{k})$ is not a matrix bialgebra with respect to the obvious basis $T$, as one needs to introduce inverse of the determinant. That can be, of course, repaired by enlarging the basis. On the other hand, $\mathcal{O}(S L(n, \mathbf{k}))$ is a matrix bialgebra and a matrix Hopf algebra with the same standard basis $T$.

The free matrix Hopf algebra $\mathcal{N G} \mathcal{L}(n, \mathbf{k})$ is the free Hopf algebra generated by the free matrix coalgebra $\mathcal{M}_{C}(n, \mathbf{k})$ (the latter is just the $\mathbf{k}$-vector space $C$ of dimension $n^{2}$ with matrix comultiplication). Equivalently, $\mathcal{N G} \mathcal{L}(n, \mathbf{k})$ is the Hopf envelope of the free matrix bialgebra $\mathcal{N} \mathcal{M}(n, \mathbf{k})$ on $n^{2}$ generators. Notice that $\mathcal{M}_{C}(n, \mathbf{k})$ is finite dimensional, $\mathcal{N} \mathcal{M}(n, \mathbf{k})$ is finitely generated and $\mathcal{N G} \mathcal{L}(n, \mathbf{k})$ is a priori not even finitely generated.

Proposition 6. Let $\Sigma_{1} \subset \Sigma_{2}$ be two sets of submatrices of the basis $T$ of $\mathcal{N M}_{n}=$ $\mathcal{N} \mathcal{M}(n, \mathbf{k})$. Then the natural maps $\Sigma_{1}^{-1} \mathcal{N} \mathcal{M}_{n} \rightarrow \Sigma_{2}^{-1} \mathcal{N} \mathcal{M}_{n}$ are 1-1.

Proof. It is known [9] that there is an algebra embedding of any free associative algebra into a skewfield $\mathcal{K}$. Let $j: \mathcal{N M}_{n} \rightarrow \mathcal{K}$ be such an embedding. By the universal property of the localization there is a unique map $j_{\Sigma}: \Sigma^{-1} \mathcal{N} \mathcal{M}_{n} \rightarrow \mathcal{K}$ provided $\Sigma$ consists of matrices which are invertible over $\mathcal{K}$. For that it is sufficient to prove that all the quasideterminants exist and they are invertible (nonzero). The quasideterminants can be defined inductively by size, provided all the involved quasiminors at every step are nonzero. In our case we deal with quasiminors of $T$ only. Their images under the projection $\mathcal{K} \rightarrow \mathcal{K} /[\mathcal{K}, \mathcal{K}]$ onto the commutative field $\mathcal{K} /[\mathcal{K}, \mathcal{K}]$ are apparently nonzero, hence they are nonzero over $\mathcal{K}$, hence invertible.

Using the universal property again, we see that the chain of natural maps $\mathcal{N} \mathcal{M}_{n} \rightarrow$ $\Sigma_{1}^{-1} \mathcal{N} \mathcal{M}_{n} \rightarrow \Sigma_{2}^{-1} \mathcal{N} \mathcal{M}_{n} \rightarrow \mathcal{K}$ composes to $j$, hence all the maps in the chain are $1-1$.

The free triangular matrix Hopf algebra $\mathcal{N B}(n, \mathbf{k})$ can be obtained directly in analogy with $\mathcal{N G} \mathcal{L}(n, \mathbf{k})$. The only difference is that the coalgebra (bialgebra) generators $t_{j}^{i}$ for $i<$ $j$ of $C=\mathcal{M}_{c}(n, \mathbf{k})(\mathcal{N} \mathcal{M}(n, \mathbf{k})$ respectively) are set to zero before taking the construction. Alternatively, one can start with $\mathcal{N G \mathcal { L }}(n, \mathbf{k})$ and consider the 2-sided ideal generated by $\left(t_{j}^{i}\right)_{r}$ for all $r=0,1,2, \ldots$ and all $i<j$. This biideal is obviously $S$-stable, hence induces a quotient map of Hopf algebras $\pi: \mathcal{N G} \mathcal{L}(n, \mathbf{k}) \rightarrow \mathcal{N B}(n, \mathbf{k})$ whose image is isomorphic to a free triangular matrix Hopf algebra. In the latter setup, we call $\mathcal{N B}(n, \mathbf{k})$, or, more precisely, the pair $(\mathcal{N B}(n, \mathbf{k}), \pi)$, the noncommutative Borel subgroup in $\mathcal{N G} \mathcal{L}(n, \mathbf{k})$.

Given a permutation $\sigma$ on $n$ letters, and an $n \times n$ matrix $G$, denote by $G^{\sigma}$ the matrix $\left(G^{\sigma}\right)_{j}^{i}=G_{j}^{\sigma(i)}$. Let $\mathcal{K}$ be the quotient skewfield of $\mathcal{G}=\mathcal{N G} \mathcal{L}(n, \mathbf{k})$. We introduce the following matrices over $\mathcal{K}$ and subalgebras of $\mathcal{K}$ :

- ${ }_{r} T$ is the copy of the matrix of generators $T \in M_{n}(C)$ in the image of $M_{n}\left(C_{r}\right)$ in $M_{n}(H(C))$, i.e. $\left({ }_{r} T\right)_{j}^{i}=\left(t_{j}^{i}\right)_{r}$. By permuting rows, we also obtain ${ }_{r} T^{\sigma}$.

- $i_{\sigma}: \mathcal{G} \rightarrow \mathcal{W}_{\sigma}$ is the Cohn invertive localization of $\mathcal{G}$ which is universal with respect to the inversion of the multiplicative set of matrices generated by all the lower right square (= principal) submatrices of ${ }_{r} T^{\sigma}$ for all $r$ even and all upper left square $(=$ coprincipal) submatrices of ${ }_{r} T^{\sigma}$ for all $r$ odd. Writing $\mathcal{G}$ is an inductive limit of Cohn localizations of $\mathcal{N} \mathcal{M}_{n}$, and representing the localization $i_{\sigma}$ by inductive limit 
too, the same argument as in the proof of Proposition 6, proves that $i$ is $1-1$ and hence $\mathcal{W}_{\sigma}$ can be viewed as a subalgebra in $\mathcal{K}$.

- The Gauss decomposition of a matrix $T=U A$ in $\mathcal{W}_{\text {id }}$ exists and is unique in an appropriate Cohn localization of $\mathcal{N M}_{n}$. The localization $i_{\text {id }}$ is 1-1, hence this decomposition implies decompositions ${ }_{r} T=U_{r} A_{r}$ if $r$ is even and ${ }_{r} T=A_{r} U_{r}$ if $r$ is odd, as matrices over $\mathcal{W}_{\text {id }}$. Here $U_{r}=\left(\left(u_{r}\right)_{j}^{i}\right)$ is an upper unitriangular and $A_{r}=\left(\left(a_{r}\right)_{j}^{i}\right)$ a lower triangular matrix. If $r$ is omitted, we mean $r=0$. This straightforwardly generalizes to $\sigma \neq \mathrm{id}$.

- Let $\mathcal{U}_{\sigma}$ be the subalgebra of $\mathcal{W}_{\sigma}$ generated by the entries of $U_{r, \sigma}$ for all $r$. Let $\mathcal{A}_{r}$ be the subalgebra of $\mathcal{W}_{\sigma}$ generated by the entries of all $A_{r, \sigma}$.

Theorem 7. 1 . $\mathcal{W}_{\sigma}$ is generated by $\mathcal{U}_{\sigma}$ and $\mathcal{A}_{\sigma}$ and the natural map $\mathcal{W}_{\sigma} \rightarrow \mathcal{K}$ is 1-1.

2. The Cohn localization map $i_{\sigma}: \mathcal{G} \rightarrow \mathcal{W}_{\sigma}$ is 1 -1 and $\mathcal{N B}(n, \mathbf{k})$-coaction compatible.

3. $b_{j}^{i} \mapsto\left(a_{\sigma}\right)_{j}^{i}$ extends to a 1-1 homomorphism of the algebras $\gamma_{\sigma}$.

4. $\gamma_{\sigma}$ is a $\mathcal{N B}(n, \mathbf{k})$-comodule map.

5. $\mathcal{U}_{\sigma}$ is a strict subalgebra of the algebra of localized coinvariants in $\mathcal{W}_{\sigma}$.

Conjecture. The set of $n$ ! Cohn localizations $i_{\sigma}: \mathcal{G} \rightarrow \mathcal{W}_{\sigma}$ covers $\mathcal{G}$.

Remarks. A) The quantum analogue (Theorems 9-11) does not follow from Theorem 7. Namely, quantum subgroups of $\mathcal{N G} \mathcal{L}(n, \mathbf{k})$ are obtained by taking big Hopf ideals, which are not a priori compatible with all the structure contained in the theorem. The quantum case is much harder, particularly for $\sigma \neq \mathrm{id}$. See below.

B) Bruhat decomposition for matrices might have some geometrical role here, but we did not consider it. Algebraically, existence of Bruhat decomposition for matrices over any skewfield is an easy classical fact [11].

Proof [78] of Theorem 7 (sketch): Perform left row operations following familiar Gauss elimination procedure, and observe that, at each step, we can extend the coaction to all the inverses which appear in the localization corresponding to that step. We introduce a block matrix $T[i]=\left(\begin{array}{ll}a & b \\ c & d\end{array}\right)$ where $i \times i$ is the size of the submatrix $d$. At the beginning of the induction procedure, $d$ has size $1 \times 1$, and $T[i]=T$ when we forget the partitioning. At each step, using left row operations, change $T[i]$ into $A[i]=\left(\begin{array}{ccc}a-b d^{-1} c & 0 \\ c & d\end{array}\right)$. Performing these left row operations amounts to the left multiplication by an upper unitriangular matrix $U[i]$. The block-entry in the left upper corner is simply the $(1,1)$-quasideterminant of the partitioned matrix $T[i]$ (observe, using heredity, which quasiminors of the underlying $n \times n$ matrix do not change in this procedure). Only the matrix $d$ has been inverted at the current step. The algebra generated by (genuine, not block) entries of $A[i]$ contains all entries of $d^{-1}$ (use invertibility of $T$ ), and it is equal to the Cohn localization of the algebra at the submatrix $d$ of the algebra at the previous step. If $P$ is the basis matrix for the noncommutative Borel subgroup $\mathcal{N B}_{n}$ (or, in a strengthened version of this argument, for the appropriate parabolic $\mathcal{N} \mathcal{P}_{n}(i)$ ), then we write $P$ as a partitoned matrix (of the same partition type as $T[i]) P=\left(\begin{array}{ll}\mathfrak{a} & 0 \\ \mathfrak{c} & \mathfrak{d}\end{array}\right)$. Then $\rho(d)=d \otimes \mathfrak{d}$, hence it is invertible in the localization tensored with $\mathcal{N B}_{n}$, because $\mathfrak{d}$ is invertible in $\mathcal{N B}_{n}$, by the simple fact that a triangular matrix is invertible iff each diagonal entry is. Coaction therefore extends to the localization. Moreover, 


$$
\rho\left(a-b d^{-1} c\right)=a \otimes \mathfrak{a}+b \otimes \mathfrak{c}-(b \otimes \mathfrak{d})\left(d^{-1} \otimes \mathfrak{d}^{-1}\right)(c \otimes \mathfrak{a}+d \otimes \mathfrak{c})=\left(a-b d^{-1} c\right) \otimes \mathfrak{a} .
$$

Hence $\rho(A[i])=A[i] \otimes P$ (matrix multiplication with $\otimes$ between the entries). This coaction is identical to the coaction on the matrix $T[i]$, hence the correspondence $\gamma^{i, 0}:(T[i])_{j}^{i} \rightarrow$ $(A[i])_{j}^{i}$ extends to an $\mathcal{N B}(n, \mathbf{k})$-comodule map on the $\mathbf{k}$-span of set of entries of $T[i]$. We start with free generators, and gradually impose relations, hence $\gamma^{i, 0}$ extends to an $\mathcal{N B}(n, \mathbf{k})$-comodule algebra map $\gamma^{i}$. Repartition $A[i]$, making $d$ of size $i+1$, call the resulting matrix $T[i+1]$, and continue the procedure. Notice that the composition of the resulting maps $\gamma^{i}$ has the same kernel as the map defining a Borel subgroup (one inclusion follows from the procedure and another from generalities on smash products). These arguments essentially show 1-4. To prove 5. just use the Gauss decomposition formula for generators of $\mathcal{U}_{\sigma}$ as a fraction of two flag quasideterminants $u_{1} u_{2}^{-1}$ and observe that $\rho_{\sigma}\left(u_{1}\right)=u_{1} \otimes b$ and $\rho_{\sigma}\left(u_{2}\right)=u_{2} \otimes b$ for the same $b \in \mathcal{N B}(n, \mathbf{k})$, and conclude that $u_{1} u_{2}^{-1}$ is a coinvariant.

14. Quantum matrix groups $[62,50,49,38,31,66]$. Let $q \in \mathbf{k}, q \neq 0$. The quantum matrix bialgebra $\mathcal{M}_{q}(n, \mathbf{k})=\mathcal{O}\left(M_{q}(n, \mathbf{k})\right)$ is the free matrix bialgebra $\mathcal{N} \mathcal{M}(n, \mathbf{k})$ with basis $T=\left(t_{\beta}^{\alpha}\right)$ modulo the smallest biideal $I$ such that the following relations hold in the quotient:

$$
\begin{array}{ll}
\alpha=\beta, \gamma<\delta \text { (same row) } & t_{\gamma}^{\alpha} t_{\delta}^{\alpha}=q t_{\delta}^{\alpha} t_{\gamma}^{\alpha}, \\
\alpha<\beta, \gamma=\delta \text { (same column) } & t_{\gamma}^{\alpha} t_{\gamma}^{\beta}=q t_{\gamma}^{\beta} t_{\gamma}^{\alpha}, \\
\alpha \neq \beta \text { and } \gamma \neq \delta & {\left[t_{\gamma}^{\alpha}, t_{\delta}^{\beta}\right]=\left(q-q^{-1}\right) t_{\gamma}^{\beta} t_{\delta}^{\alpha}(\theta(\delta>\gamma)-\theta(\alpha>\beta)),}
\end{array}
$$

where $\theta$ (true) $=1, \theta$ (false) $=0$, and [,] stands for the ordinary commutator.

$\mathcal{M}_{q}(n, \mathbf{k})$ is a domain. There are other versions, including the multiparametric case $\mathcal{M}_{P, Q}(n, \mathbf{k})$, cf. $[51,85,76]$. Some of the results below generalize to $\mathcal{M}_{P, Q}(n, \mathbf{k})$.

The quantum determinant $D \in \mathcal{M}_{q}(n, \mathbf{k})$ is defined by any of the formulas

$$
D=\sum_{\sigma \in \Sigma(n)}(-q)^{l(\sigma)-l(\tau)} t_{\sigma(1)}^{\tau(1)} t_{\sigma(2)}^{\tau(2)} \cdots t_{\sigma(n)}^{\tau(n)}=\sum_{\sigma \in \Sigma(n)}(-q)^{l(\sigma)-l(\tau)} t_{\tau(1)}^{\sigma(1)} t_{\tau(2)}^{\sigma(2)} \cdots t_{\tau(n)}^{\sigma(n)},
$$

where $\Sigma(n)$ is the permutation group on $n$ letters and $l$ the standard length function on $\Sigma(n)$ and $\tau \in \Sigma(n)$ is a fixed permutation (say identity), the choice being irrelevant.

$D$ is a central element in $\mathcal{M}_{q}(n, \mathbf{k}) . D$ can alternatively be defined using canonical coactions on the quantum exterior algebra $[62,76]$.

Selecting $m$ rows and columns out of $n$ clearly singles out a subalgebra in $\mathcal{M}_{q}(n, \mathbf{k})$ isomorphic to $\mathcal{M}_{q}(m, \mathbf{k})$. Quantum minors are the corresponding quantum determinants $D_{L}^{K}$ for the selected row and column $m$-multilabels $K$ and $L$. Quantum minors do not necessarily commute with elements which do not belong to the subalgebra $\mathcal{M}_{q}(m, \mathbf{k})$ generated by selected entries of $T_{L}^{K}$. Numerous authors found, often independently, a large number of useful commutation relations, identities and computational principles involving quantum minors, cf. e.g. $[62,31,41,76]$. The starting point is

LAPLACE EXPANSION. For any pair of ordered $m$-multiindices $K, L$ (labels: 1 to $n$ ),

$$
\begin{aligned}
\delta_{L}^{K} D & =\sum_{J}(-q)^{J-L} D_{J}^{K} D_{\hat{J}}^{\hat{L}}=\sum_{J}(-q)^{J-L} D_{K}^{J} D_{\hat{L}}^{\hat{J}} \\
& =\sum_{J}(-q)^{L-J} D_{\hat{J}}^{\hat{L}} D_{J}^{K}=\sum_{J}(-q)^{L-J} D_{\hat{L}}^{\hat{J}} D_{K}^{J}
\end{aligned}
$$


where $J$ runs over $m$-multiindices; the name of a multiindex in exponent denotes the sum of labels, and ^denotes the ordered complement with respect to the set of labels $\{1, \ldots, n\}$.

Define $\mathcal{S} \mathcal{L}_{q}(n, \mathbf{k})=\mathcal{O}\left(S L_{q}(n, \mathbf{k})\right)=\mathcal{M}_{q}(n, \mathbf{k}) / L$ where $L$ is the biideal generated by $(D-1)$. Similarly define $\mathcal{G} \mathcal{L}_{q}(n, \mathbf{k})=\mathcal{O}\left(G L_{q}(n, \mathbf{k})\right)$ as the localization of $\mathcal{M}_{q}(n, \mathbf{k})$ at the central element $D$. In the multiparametric case $\mathcal{G} \mathcal{L}_{P, Q}(n, \mathbf{k})$ is more interesting than $\mathcal{S} \mathcal{L}_{P, Q}(n, \mathbf{k})$ because the latter typically degenerates. $\mathcal{S} \mathcal{L}_{q}(n, \mathbf{k})$ and $\mathcal{G L}_{q}(n, \mathbf{k})$ are Hopf algebras. The following formula for the antipode on the generators is forced by Laplace expansion formulas:

$$
S t_{j}^{i}=(-q)^{i-j} D^{-1} D_{\hat{i}}^{\hat{j}}
$$

Antipode gives the inverse of a matrix $T$ in the matrix ring over $\mathcal{G L}_{q}(n, \mathbf{k})$ by $S t_{j}^{i}=$ $\left(T^{-1}\right)_{j}^{i}$ and this, in turn, gives formulas for all the quasideterminants of $T$. Namely, $|G|_{i j}^{-1}=\left(G^{-1}\right)_{j i}$ whenever both sides are defined. Hence

$$
|T|_{i j}=\left(S\left(t_{i}^{j}\right)\right)^{-1}=(-q)^{j-i} D\left(D_{\hat{j}}^{\hat{i}}\right)^{-1} .
$$

Thus one can use all we know about quasideterminants to study quantum determinants, and in particular one can write down formulas for Gauss decomposition of a matrix $T$ and matrices obtained from $T$ by permutation of rows in terms of quantum determinants.

THEOREM 8 [76]. Every set of quantum minors multiplicatively generates a 2-sided Ore set in $\mathcal{M}_{q}(n, \mathbf{k})$. This Ore localization is isomorphic to Cohn localization at the set of corresponding submatrices of $T$. The localization map is 1-1. An analogue holds for $\mathcal{G} \mathcal{L}_{q}(n, \mathbf{k}), \mathcal{S} \mathcal{L}_{q}(n, \mathbf{k})$ and for (strong) multiparametric deformations $\mathcal{M}_{P, Q}(n, \mathbf{k})$ and $\mathcal{G} \mathcal{L}_{P, Q}(n, \mathbf{k})$.

The proof is easy when, for each of the quantum minors involved, the selected rows are adjacent (without gaps) and the selected columns of $T$ are adjacent too. The general case (with gaps) has been proved [76] using several reductions to special cases and an induction using rather nontrivial commutation relations involving quantum minors. "Strong" denotes a usually imposed condition on deformation parameters $[1,15,76]$.

From now on, $\mathcal{G}$ will denote either $\mathcal{G} \mathcal{L}_{q}(n, \mathbf{k})$ or $\mathcal{S} \mathcal{L}_{q}(n, \mathbf{k})$.

The quantum Borel subgroup $\mathcal{B}=\mathcal{B}_{q}(n, \mathbf{k})$ is the quotient of $\mathcal{G}$ by the biideal $I$ generated by $t_{j}^{i}$ with $i<j . I$ is a Hopf ideal, hence $\mathcal{B}$ is a Hopf algebra.

Let $I$ be a subset $I \subset\{1, \ldots, n-1\}$ and $I_{1}, \ldots, I_{m}$ be its connected components ordered in such a way that $i<j$ implies every element of $I_{i}$ is smaller than every element of $I_{j}$. Denote $I_{k}^{+}=I_{k} \cup\left\{i+1: i \in I_{k}\right\}$ for all $1 \leq k \leq m$. Then the ideal $J_{I}$ generated by $t_{j}^{i}$ where $i<j$ and $(i, j) \notin \cup_{k} I_{k}^{+} \times I_{k}^{+}$is a Hopf ideal. If $I$ is nonempty, the quotient Hopf algebra $\mathcal{G} / J_{I}$ is called the quantum parabolic subgroup $\mathcal{P}^{I}$. For $I=\emptyset$ we obtain $\mathcal{P}^{I}=\mathcal{B}$ and for $I=\{1, \ldots, n-1\}$ we obtain $\mathcal{P}^{I}=\mathcal{G}$. Notice that $I_{k}^{+} \times I_{k}^{+}$is the set of labels of all boxes in a rectangle with two of the corners at the main diagonal. Hence there is a natural partition of $T$ into square submatrices such that the anihilated ideal is generated by the elements in the strictly upper block triangular part of $T$. The dimensions of the blocks are determined by the combinatorics of the set $I$. For a given ring $R$, equip the matrices in the matrix ring $M_{n}(R)$ with that same block partition, and let $M_{n}^{I}(R)$ be the resulting ring of block-matrices. The identity map surely gives the ring isomorphism between $M_{n}(R)$ and $M_{n}^{I}(R)$, but the notions like upper triangular, unidiagonal etc. differ. 
For $I=\emptyset$ we obtain $\mathcal{P}=\mathcal{B}$ and for $I=\{1, \ldots, n-1\}$ we obtain $\mathcal{P}=\mathcal{G}$.

If $I^{\prime} \subset I$ then $J_{I} \subset J_{I^{\prime}}$, hence there is a natural Hopf algebra surjection $\pi_{I, I^{\prime}}: \mathcal{P}^{I} \rightarrow$ $\mathcal{P}^{I^{\prime}}$ generalizing $\pi: \mathcal{G} \rightarrow \mathcal{B}$ and $\pi_{I}: \mathcal{G} \rightarrow \mathcal{P}^{I}$.

For a permutation $\sigma \in \Sigma(n)$, let $w_{\sigma}$ be the corresponding permutation matrix, i.e. for every matrix $G$ we have $\left(w_{\sigma}^{-1} G\right)_{j}^{i}=g_{j}^{\sigma(i)}$. Consider now the noncommutative Gauss decomposition in $M_{n}^{I^{\prime}}\left(\mathcal{K}\left(\mathcal{P}^{I}\right)\right)$, where $\mathcal{K}\left(\mathcal{P}^{I}\right)$ is the quotient skewfield of $\mathcal{P}^{I}$ :

$$
\pi_{I}(T)=w_{\sigma} U_{\sigma} A_{\sigma}
$$

where $U_{\sigma}$ is upper unitriangular and $A_{\sigma}$ lower triangular in $M_{n}^{I}\left(\mathcal{K}\left(\mathcal{P}^{I}\right)\right)$, i.e. in the sense of $I^{\prime}$-blocks.

Let $\left(u_{\sigma}\right)_{j}^{i}$ be the $(i, j)$-th entry of $U_{\sigma}$, but now taken as a matrix without block partition and similarly for $\left(a_{\sigma}\right)_{j}^{i}$.

For a subset $X$ in some algebra, denote by $\langle X\rangle$ the subalgebra generated by $X$. Denote

$$
\begin{aligned}
& \mathcal{U}_{\sigma}=\left\langle\left(u_{\sigma}\right)_{j}^{i}\right\rangle \subset \mathcal{K}\left(\mathcal{P}^{I}\right), \\
& \mathcal{A}_{\sigma}=\left\langle\left(a_{\sigma}\right)_{j}^{i}\right\rangle \subset \mathcal{K}\left(\mathcal{P}^{I}\right), \\
& \mathcal{W}_{\sigma}=\left\langle\mathcal{U}_{\sigma}, \mathcal{A}_{\sigma}\right\rangle \subset \mathcal{K}\left(\mathcal{P}^{I}\right) .
\end{aligned}
$$

The permutation group $\Sigma(n)$ plays here a role of the Weyl group of $S L_{n}$. If we consider only the case of the Borel subgroup, then the different permutations in $\Sigma(n)$ give different subalgebras $\mathcal{U}_{\sigma}$ in $\mathcal{G}$. If we consider other parabolics, those permutations for which the notion of being upper $I^{\prime}$-block triangular agrees, lead only to permutations among the generators $\left(u_{\sigma}\right)_{j}^{i}$ and hence yield identical subalgebras in $\mathcal{G}$. Thus for general parabolics one may consider the relative Weyl group to avoid repetitions.

Equation (13) can be solved in terms of quasideterminants, applying formulas for noncommutative Gauss decomposition to $G=w_{\sigma}^{-1} \pi_{I}(T)$ considered as a matrix in $M_{n}^{I^{\prime}}\left(\mathcal{K}\left(\mathcal{P}^{I}\right)\right)$. The general case is somewhat cumbersome. The simplest, Borel case, as well as the decomposition where the Hopf ideals are generated by all entries in the upper triangular part of first several rows ("fine Gauss decomposition"), were treated in some detail in the author's thesis. We are now going to formulate the corresponding structure theorems for the Borel case (i.e. analogue of $G / B$ ) which give data for a locally trivial quantum principal fibration in the sense developed in the earlier sections of this paper.

TheOREM 9 [76, 78]. Let $S_{\sigma}$ be the Ore set in $\mathcal{G}=\mathcal{G L}_{q}(n)$ or $\mathcal{G}=\mathcal{S L}_{q}(n)$ generated by all principal (= right lower corner) quantum minors of $w_{\sigma}^{-1} T$.

(i) The natural coaction

$$
\rho_{\mathcal{B}}:(\mathrm{id} \otimes \pi) \circ \Delta_{\mathcal{G}}: \mathcal{G} \rightarrow \mathcal{G} \otimes \mathcal{B}
$$

extends to localization making it a $\mathcal{B}$-comodule algebra, i.e. $S$ is $\rho_{\mathcal{B}}$-compatible.

(ii) The quantum Gauss decomposition

$$
T=w_{\sigma} U_{\sigma} A_{\sigma}
$$

where $U_{\sigma}$ is upper unitriangular and $A_{\sigma}$ lower triangular has a unique solution for $U_{\sigma}$ and $A_{\sigma}$ in matrices over the localized ring $S_{\sigma}^{-1} \mathcal{G}$.

(iii) The natural map of $S_{\sigma}^{-1} \mathcal{G}$ into the quotient skewfield identifies $S_{\sigma}^{-1} \mathcal{G}$ with $\mathcal{W}_{\sigma}$. 
Theorem $10[76,78]$. Let $b_{j}^{i}=\pi\left(t_{j}^{i}\right)$ be the $(i, j)$-th generator of quantum Borel. The correspondence $b_{j}^{i} \rightarrow\left(a_{\sigma}\right)_{j}^{i}$ extends to a homomorphism of $\mathcal{B}$-comodule algebras

$$
\gamma_{\sigma}: \mathcal{B} \rightarrow S_{\sigma}^{-1} \mathcal{G}
$$

This theorem is relatively easy to check for $\sigma=$ id, i.e. for the main cell. It is harder to show (algebra homomorphism part) for other $\sigma$, as it amounts to showing that $\left(a_{\sigma}\right)_{j}^{i}$, which are, up to constants, ratios of quantum minors, satisfy the relations which $b_{j}^{i}$ do. There is however a principle ("included row exchange principle" [76]) which enables one to transfer certain identities among the quantum minors to identities with rows systematically permuted. Using this principle, Theorem 10 reduces to the case $\sigma=\mathrm{id}$. In the case $\sigma=$ id there is an alternative proof. Namely, one can use row operations starting with the matrix $T$, performing a version of Gauss elimination procedure which ends with a triangular matrix $\left(\left(a_{\mathrm{id}}\right)_{j}^{i}\right)$. One simply shows that at every step the required identities among the entries are preserved modulo the ideal ker $\pi$. However, this "natural" argument does not apply to the case $\sigma \neq \mathrm{id}$. Namely, in that case, we start with the matrix $w_{\sigma}^{-1} T$ instead. Its entries do not satisfy the required identities modulo ker $\pi$. The row operations in that case change the identities too. In fact, the situation gets improved, and we end with the correct identities! It works but a better explanation ought to be found.

TheOREM $11[76,78]$. (i) $\mathcal{U}_{\sigma} \subset\left(S_{\sigma}^{-1} \mathcal{G}\right)^{\mathrm{co} \mathcal{B}}$.

(ii) If $\sigma=\mathrm{id}$ or if $\sigma=(n \ldots 21)$ then $\mathcal{U}_{\sigma}=\left(S_{\sigma}^{-1} \mathcal{G}\right)^{\mathrm{co} \mathcal{B}}$.

Assertion (i) follows by a direct and simple computation after expressing $\left(u_{\sigma}\right)_{j}^{i}$ in terms of quantum minors. By Theorem 9 (iii) we see that $\mathcal{A}=\gamma_{\sigma}(\mathcal{B})$ and $\mathcal{U}_{\sigma}$ generate $S_{\sigma}^{-1} \mathcal{G}$. Hence (i) implies by standard arguments (cf. our discussion of higher smash products above) that in order to prove (ii) it is sufficient to show that $\mathcal{U}_{\sigma}$ is invariant with respect to the $\mathcal{B}$-action

$$
b \triangleright u=\sum \gamma_{\sigma}\left(b_{(1)}\right) u \gamma_{\sigma}\left(S b_{(2)}\right)
$$

Furthermore, it is enough to show this on generators, i.e. that

$$
b_{j}^{i} \triangleright\left(u_{\sigma}\right)_{l}^{k}=\sum_{i \geq s \geq j} \gamma_{\sigma}\left(b_{s}^{i}\right)\left(u_{\sigma}\right)_{l}^{k} \gamma_{\sigma}\left(S b_{j}^{s}\right) \in \mathcal{U}_{\sigma} .
$$

We know that $a_{j}^{i}=\sigma\left(b_{j}^{i}\right)$; we can also express $a_{j}^{i}$ in terms of quasideterminants (formula for Gauss decomposition!) and then reexpress in terms of quantum determinants. Here $S b_{j}^{l} \equiv S_{\mathcal{B}}\left(b_{j}^{l}\right)$ is equal to $\pi\left(S_{\mathcal{G}} t_{j}^{l}\right)$ and hence, up to a scalar, to the projection in $\mathcal{B}$ of a certain quantum (cofactor) minor. Thus $\gamma_{\sigma}\left(S b_{j}^{s}\right)$ is also a quantum minor expression but in the algebra $\mathcal{A}_{\sigma}$. If they commuted, say up to a constant factor, $\left(a_{\sigma}\right)_{s}^{i}$ with $\left(u_{\sigma}\right)_{l}^{k}$, we would obtain a scalar factor times

$$
\left(u_{\sigma}\right)_{l}^{k} \sum_{s} \gamma_{\sigma}\left(b_{s}^{i}\right) \gamma_{\sigma}\left(S b_{j}^{s}\right)=\delta_{j}^{i} u_{l}^{k}
$$

where $\delta_{j}^{i}$ is the Kronecker delta. In other words, if $\left(a_{\sigma}\right)_{s}^{i}$ and $\left(u_{\sigma}\right)_{l}^{k}$ commute up to a number depending only on $i, j, k, l$, we obtain an answer proportional to one obtained from a trivial action $b . u=\epsilon(b) u$. A natural strategy is hence to try commuting $\left(a_{\sigma}\right)_{s}^{i}$ (or alternatively, the other, antipode part) with $\left(u_{\sigma}\right)_{l}^{k}$. The result depends on relative 
positions of indices $i, j, k, l$ and it implies Theorem 11 (ii), which has been checked case by case in the author's thesis.

Assertion (ii) is not a sharp result: we need the exhaustive list of all $n$ and $\sigma \in$ $\Sigma(n)$ when the statement holds. The case by case proof is brute force. For general $\sigma$ extra summands appear which seem not to live in $\mathcal{U}_{\sigma}$. This phenomenon needs further clarification.

15. Applications. Comparison with other approaches. The concept of a family of Perelomov coherent states can be generalized for Hopf algebras [76, 79] in the framework of this paper. We believe that in the special case of quantum groups the coherent states of [36] essentially coincide with our construction. Under rather general assumptions a Hopf algebraic analogue of a classical resolution of unity by coherent states has been proved by the author in [79].

In the approaches to quantum principal bundles without localization, noncommutative analogues of the differential calculus and of connections on fiber bundles were considered in many earlier works, e.g. [7, 63, 29]. V. Lunts and A. Rosenberg [44, 43] properly extended Grothendieck's definition [27] of the rings of regular differential operators to noncommutative rings and considered extensibility of Hopf module algebra actions given by the regular differential operators. For a left Ore set $S$ in a domain $R$, a derivation $d: R \rightarrow R$ always uniquely extends $[16,44]$ to a derivation $d_{S}: S^{-1} R \rightarrow S^{-1} R$. Proving that fact is a good exercise [77] for a newcomer to Ore localizations. If a derivation $d: R \rightarrow \Omega^{1}(R)$ takes values in an $R$ - $R$-bimodule $\Omega^{1}(R)$ such that $\left(d, \Omega^{1}(R)\right)$ define a 1 st order differential calculus, then the same proof produces a unique extension $d_{S}: S^{-1} R \rightarrow$ $S^{-1} R \otimes_{R} \Omega^{1}(R)$ such that $\left(d_{S}, S^{-1} R \otimes_{R} \Omega^{1}(R)\right)$ is again a 1 st order differential calculus, provided an additional condition is satisfied, which we may call the differential left Ore condition. Namely, $\forall t \in S, \forall r \in R, \exists s \in S$ and $\exists \omega \in \Omega^{1}(R)$, such that $s d r=\omega t$.

We are interested in gluing locally defined differential calculi over the quotient spaces, and in the comparison of the connections defined in quantum vector bundles over different local charts. For that purpose, a version of gauge transformations of [7] will play a role.

For a rather different concept of local triviality of noncommutative principal bundles see [30] and references therein. An early notion of locally trivial vector bundle using Gabriel localizations appeared in [72](Appendix 2). Sheaf-theoretic ideas and localization were present much earlier in noncommutative geometry, namely in the study of sheaves over noncommutative spectra [57, 90, 26].

If $q$ is a primitive root of unity, $\mathcal{S} \mathcal{L}_{q}(n, \mathbb{C})$ has a large center. This enables an alternative approach [67] to $\mathcal{S L}_{q}(n, \mathbb{C})$ using small noncommutative sheaves over ordinary $S L(n, \mathbb{C})$. In that case the centers of our localized algebras $\mathcal{W}_{\sigma}$ are also big, which enables a detailed comparison of the two approaches.

Quantum flag varieties can be defined and studied using the representation theory of quantized enveloping algebras [82, 39, 42, 45, 35] or by an Ansatz exploring quantum minors [86]. In these approaches, the quantum flag varieties are described by a noncommutative graded ring $\mathcal{F}_{q}$, or by an appropriate quotient of the category of (multi)graded $\mathcal{F}_{q}$-modules. In classical limit $q=1$, this ring can be viewed as the ring of homogeneous functions on the projective variety $G / B$, or as a ring of regular functions on the 
quasiaffine variety $G / U$ where $U$ is the unipotent radical of $B$. For type $\mathrm{A}, \operatorname{ring} \mathcal{F}_{q}$ is generated by quantum flag minors. In our conventions, quantum flag minors are those quantum $m$-minors which always include all columns from $n-m+1$ to $n$. The algebra of the main unshifted cell in our construction can be obtained by taking the zero graded part $\left(\tilde{S}^{-1} \mathcal{F}_{q}\right)_{0}$ of the localization of the algebra $\mathcal{F}_{q}$ at the homogeneous Ore set $\tilde{S}$ generated by the principal flag minors. Several authors introduced the quantum flag and Grassmann varieties, working only on the main cell [73, 36, 84].

We note that the Weyl group shifts $\mathcal{W}_{\sigma}$ are pairwise manifestly isomorphic for $q=1$ but, in general, nonisomorphic for $q \neq 1$. It would be interesting, to complete the picture, to find out whether they are Morita equivalent.

Quantum partial flag varieties, and Grassmannians in particular, can be approached by the methods of this paper. Simple refinements of the quantum Gauss decomposition play a role there (as parabolics correspond to block triangular matrices). This enables induction over stages and provides examples of quantum fibre bundles between different partial flag varieties corresponding to inclusions of parabolics.

However, we expect the example $\mathcal{N} \mathcal{G} \mathcal{L}_{n} / \mathcal{N B}_{n}$ to be more important, as the quantum flag varieties seem to be already pretty tamed by other approaches, and the fully noncommutative case opens door to a more unknown area. We also believe in the importance of the general foundational questions on quotients, coaction-compatible localizations and cohomology. I hope that a reader of this paper could observe a number of obvious promising open questions which the localization approach to quotients opens up.

Acknowledgements. I warmly thank Prof. Joel Robbin for his interest and advice during preparation of my Ph.D. thesis. Several friends, colleagues and teachers were nice to encourage me already early in this project, including I. Žutić, G. Benkart, B. Balantekin, S. Witherspoon, A. Chubukov. Prof. D. Passman scheduled me for a ring theory seminar in Spring 1998, where the first promising result, Corollary 4 of this paper and an $S L_{q}(2)$ version of Theorems 9-11 were presented. I thank him and other people who scheduled or attended my seminars in Madison, West Lafayette, Zagreb and Bloomington. Finally, during the last year, I had a chance to share wider written, phone and oral communication on the topic of this work and I thank all who have had patience to listen or read, and to point out various advice, warnings, language errors, literature remarks etc. Among them A. Ram and C. Goebel suggested numerous improvements in the thesis text, and I. Mori in an after-thesis preprint. I thank A. Rosenberg for sharing insights in a phone conversation, and A. Voronov à propos the root of unity case. Y. Soibelman suggested a direction for further applications. Most recently, V. Lunts advised me to rework the notion of quantum coset space via cosimplicial techniques (work in progress). I am thankful to the organizers of the Warsaw conference "Noncommutative Geometry and Quantum Groups" (2001) for providing a nice workshop atmosphere and reserving a time slot for my presentation; similarly to the organizers of "International Hopf Algebra Conference" at De Paul University (2002). I am grateful to H.-J. Schneider for referring me to $[58,85]$. Thanks to my numerous conversants from the two events, for their interest and encouragement, particularly to S. Majid. I thank the editor and the referee for the useful suggestions. Most of the new results in this paper were obtained during my Ph.D. 
study at the University of Wisconsin-Madison and the paper has been completed during my stay at Purdue University.

\section{References}

[1] M. Artin, W. Schelter and J. Tate, Quantum deformations of $G L(n)$, Comm. Pure Appl. Math. 44 (1991), 879-895.

[2] M. Barr and C. Wells, Toposes, Triples and Theories, Grund. Math. Wiss. 178, Springer, 1985.

[3] J. M. Beck, Triples, algebras and cohomology, Ph.D. Thesis, Columbia University, 1963.

[4] F. Borceux, Handbook of Categorical Algebra, 3 vols., Enc. of Math. and its Appl. 50, Cambridge Univ. Press, 1994.

[5] A. Borel, Linear Algebraic Groups, 2nd edition, GTM 126, Springer, 1991.

[6] J. Bernstein and V. Lunts, Equivariant Sheaves and Functors, Lecture Notes in Mathematics 1578, Springer, Berlin, 1994.

[7] T. Brzeziński and S. Majid, Quantum group gauge theory on quantum spaces, Comm. Math. Phys. 157 (1993), 591-638.

[8] A. Connes, Noncommutative Geometry, Academic Press, New York, 1994.

[9] P. M. Cohn, Free Rings and Their Relations, Academic Press, 1971.

[10] P. M. Cohn, Inversive localization in Noetherian rings, Comm. Pure Appl. Math. 26 (1973), 679-691.

[11] P. M. Cohn, Algebra, Vol. 3, 2nd ed., John Wiley and Sons, Chichester, 1991.

[12] P. Deligne, Catégories tannakiennes, The Grothendieck Festschrift, Vol. II, Progr. Math. 87, Birkhäuser, 1990, 111-195.

[13] P. Deligne, Théorie de Hodge III, Publ. Math. Inst. Hautes Études Sci. 44 (1974), 5-77.

[14] M. Demazure and P. Gabriel, Introduction to Algebraic Geometry and Algebraic Groups, North-Holland Math. Studies 39, North-Holland, 1980.

[15] E. E. Demidov, Multiparameter quantum deformations of the group GL( $n)$, Uspehi Mat. Nauk 46 (1991), no. 4(280), 147-148 (in Russian); translation in Russian Math. Surveys 46 (1991), 169-171.

[16] J. Dixmier, Enveloping Algebras, North-Holland, Amsterdam, 1977 (new version: AMS, 1997).

[17] V. G. Drinfel'd, Quantum groups, in: Proc. ICM-86, 798-820.

[18] V. P. Elizarov, Rings of quotients, Algebra i Logika 8 (1969), 381-424 (in Russian).

[19] P. Gabriel, Des catégories abéliennes, Bull. Soc. Math. France 90 (1962), 323-448.

[20] P. Gabriel and M. Zisman, Calculus of Fractions and Homotopy Theory, Springer, 1967.

[21] S. I. Gelfand and Yu. I. Manin, Methods of Homological Algebra, Springer, 1996; translated from the Russian original, Nauka, 1988.

[22] I. M. Gel'fand and V. S. Retakh, Determinants of matrices over noncommutative rings, Funct. Anal. Appl. 25 (1991), no. 2, 91-102 (in Russian); transl. 21 (1991), 51-58.

[23] I. M. Gel'fand and V. S. Retakh, A theory of noncommutative determinants and characteristic functions of graphs, Funct. Anal. Appl. 26 (1992), no. 4, 231-246.

[24] I. M. Gel'fand and V. S. Retakh, Quasideterminants I, Selecta Math., New Series 3 (1997), 517-546; q-alg/9705026.

[25] J. Giraud, Cohomologie non abélienne, Grundlehren Math. Wiss. 179, Springer, 1971. 
[26] J. S. Golan, J. Raynaud and F. van Oystaeyen, Sheaves over the spectra of certain noncommutative rings, Comm. Alg. 4 (1976), 491-502.

[27] A. Grothendieck, Éléments de géométrie algébrique. IV. Étude locale de schémas et de morphismes de schémas, Publ. Math. IHES 32 (1967).

[28] A. Grothendieck et al., Revêtements étales et groupe fondamental, in: Séminaire de Géométrie Algébrique du Bois Marie 1960-1961 (SGA 1), LNM 224, Springer, 1971, retyped as: arXiv:math.AG/0206203.

[29] P. Hajac, Strong connections on quantum principal bundles, Comm. Math. Phys. 182 (1996), 579-617.

[30] P. Hajac, R. Matthes and W. Szymański, Locally trivial quantum Hopf fibration, arXiv:math.QA/0112317.

[31] M. Hashimoto and T. Hayashi, Quantum multilinear algebra, Tôhoku Math J. 44 (1992), 471-521.

[32] J. C. Jantzen, Representations of Algebraic Groups, Pure and Appl. Math. 131, Academic Press, 1987.

[33] J. F. Jardine, Stacks and the homotopy theory of simplicial sheaves, Equivariant Stable Homotopy Theory and Related Areas (Stanford, CA, 2000), Homology Homotopy Appl. 3 (2001), 361-384 (electronic).

[34] P. Jara, A. Verschoren and C. Vidal, Localization and Sheaves: a Relative Point of View, Pitman Res. Notes in Math. 339, Longman 1995.

[35] A. Joseph, Quantum Groups and their Primitive Ideals, Springer, 1995.

[36] B. Jurčo and P. Štovíček, Coherent states for quantum compact groups, Comm. Math. Phys. 182 (1996), 221-251.

[37] K. H. Kamps and T. Porter, Abstract Homotopy and Simple Homotopy Theory, World Scientific, 1997.

[38] A. U. Klymik and K. Schmüdgen, Quantum Groups and Their Representations, Springer, 1997.

[39] L. I. Korogodski and Ya. S. Soibelman, Algebras of Functions on Quantum Groups I, Math. Surveys and Monographs 56, AMS, 1998.

[40] M. Kontsevich and A. L. Rosenberg, Noncommutative smooth spaces, in: The Gelfand Mathematical Seminars, 1996-1999, Birkhäuser Boston, 2000, 85-108; also arXive: math.AG/9812158.

[41] D. Krob and B. Leclerc, Minor identities for quasi-determinants and quantum determinants, Comm. Math. Phys. 169 (1995), 1-23.

[42] V. Lakshmibai and N. Reshetikhin, Quantum flag and Schubert schemes, in: Deformation Theory and Quantum Groups with Applications to Mathematical Physics (Amherst, MA, 1990), Contemp. Math. 134, AMS, 1992, 145-181.

[43] V. A. Lunts and A. L. Rosenberg, Differential calculus in noncommutative algebraic geometry I. D-calculus on noncommutative rings, Max Planck Institute preprint MPI 96-53, Bonn, 1996.

[44] V. A. Lunts and A. L. Rosenberg, Differential operators on noncommutative rings, Selecta Math. (N.S.) 3 (1997), 335-359.

[45] V. A. Lunts and A. L. Rosenberg, Localization for quantum groups, Selecta Math. (N.S.) 5 (1999), 123-159.

[46] V. Lunts and Z. Škoda, work in progress.

[47] S. Mac Lane, Categories for the Working Mathematician, GTM 5, Springer, 1971.

[48] S. Mac Lane and I. Moerdijk, Sheaves in Geometry and Logic, Springer, 1992. 
[49] S. Majid, Foundations of Quantum Group Theory, Cambridge University Press, 1995.

[50] Yu. I. Manin, Quantum Groups and Non-Commutative Geometry, CRM, Montreal, 1988.

[51] Yu. I. Manin, Multiparametric quantum deformation of the general linear supergroup, Comm. Math. Phys. 123 (1989), 163-175.

[52] A. Masuoka, Quotient theory of Hopf algebras, in: Advances in Hopf Algebras (Chicago, IL, 1992), Lec. Notes in Pure and Appl. Math. 158, Dekker, NY, 1994, 107-133.

[53] J. S. Milne, Étale Cohomology, Princeton Mathematical Series 33, 1980.

[54] I. Moerdijk, Classifying Spaces and Classifying Topoi, LNM 1616, Springer, 1995.

[55] I. Moerdijk, The classifying topos of a continuous groupoid I, Trans. AMS 310 (1998), 629-668.

[56] S. Montgomery, Hopf Algebras and Their Actions on Rings, CBMS Regional Conference Series in Mathematics 82, AMS, 1993.

[57] D. C. Murdoch and F. van Oystaeyen, Noncommutative localization and sheaves, J. Alg. 38 (1975), 500-515.

[58] S. Montgomery and H.-J. Schneider, Hopf crossed products, rings of quotients and prime ideals, Adv. Math. 112 (1995), 1-55.

[59] J. Ndirahisha and F. van Oystaeyen, Grothendieck representations of categories and canonical noncommutative topologies, K-Theory 25 (2002), 355-371.

[60] P. Nuss, Noncommutative descent and non-abelian cohomology, K-Theory 12 (1997), 2374.

[61] O. Ore, Linear equations in noncommutative fields, Ann. Math. 32 (1931), 463-477.

[62] B. Parshall and J. Wang, Quantum linear groups, Memoirs AMS 439 (1991).

[63] M. J. Pflaum and P. Schauenburg, Differential calculi on noncommutative bundles, qalg/9612030.

[64] N. Popescu, Abelian Categories with Applications to Rings and Modules, London Math. Soc. Monographs 3, Academic Press, 1973.

[65] B. Requejo, Topological localization in Fréchet algebras, J. Math. Anal. Appl. 189 (1995), 160-178.

[66] N. Yu. Reshetikhin, L. A. Takhtajan and L. D. Faddeev, Quantization of Lie groups and Lie algebras, Algebra i Analiz 1 (1989), 178 (in Russian), English translation in Leningrad Math. J. 1.

[67] N. Reshetikhin, A. Voronov and A. Weinstein, Semiquantum geometry, Algebraic Geometry 5, J. Math. Sci. 82 (1996), 3255-3267; q-alg/9606007.

[68] A. L. Rosenberg, Noncommutative local algebra, Geom. Funct. Anal. 4 (1994), 545-585.

[69] A. L. Rosenberg, Noncommutative schemes, Comp. Math. 112 (1998), 93-125.

[70] A. L. Rosenberg, Noncommutative spaces and schemes, Max Planck Institute preprint MPI-1999-84, Bonn 1999.

[71] A. L. Rosenberg, Noncommutative Algebraic Geometry and Representations of Quantized Algebras, MAIA 330, Kluwer, 1995.

[72] A. L. Rosenberg, Non-commutative affine semischemes and schemes, Seminar on supermanifolds No. 26, edited by D. Leites, Dept. of Math., Univ. of Stockholm, 1988.

[73] H. Sazdjian, Y. S. Stanev, and I. T. Todorov, SU(3)-coherent state operators and invariant correlation functions and their quantum group counterparts, J. Math. Phys. 36 (1995), 2030-2052.

[74] H. J. Schneider, Lectures on Hopf Algebras, Trabajos de Matemática 31, Universidad Nacional de Córdoba, 1995. 
[75] H.-J. Schneider, Principal homogeneous spaces for arbitrary Hopf algebras, Israel J. Math. 72 (1990), 167-195.

[76] Z. Škoda, Coset spaces for quantum groups, Ph.D. Thesis, Univ. of Wisconsin-Madison, Jan. 2002 (available on request).

[77] Z. Škoda, Localized coinvariants I, preprint.

[78] Z. Škoda, Localized coinvariants II, in preparation.

[79] Z. Škoda, Coherent states for Hopf algebras, preprint; math.QA/0303357.

[80] Z. Škoda, Lectures on Ore localizations and localizations in noncommutative geometry, preprint.

[81] P. S. Smith, Non-commutative algebraic geometry, preprint lecture notes at Univ. of Washington, 2000 .

[82] Ya. S. Soibelman, On the quantum flag manifold, Funct. Anal. Appl. 26 (1992), 225-227.

[83] B. Stenström, Rings of Quotients, Grundlehren Math. Wiss. 217, Springer, 1975.

[84] P. Štovíček, Quantum Grassmann manifolds, Comm. Math. Phys. 158 (1993), 135-153.

[85] B. Strüber, Multiparameter quantum matrices over skew fields, J. Alg. 208 (1998), 551-574.

[86] E. J. Taft and J. Towber, Quantum deformation of flag schemes and Grassmann schemes I. A q-deformation of the shape-algebra for GL(n), J. Alg. 142 (1991), 1-36.

[87] M. Takeuchi, Free Hopf algebras generated by coalgebras, J. Math. Soc. Japan 23 (1971), 561-582.

[88] M. Takeuchi, Matric bialgebras and quantum groups, Israel J. Math. 72 (1990), 232-251.

[89] F. van Oystaeyen, Algebraic Geometry for Associative Algebras, Marcel Dekker, 2000.

[90] F. van Oystaeyen and A. Verschoren, Reflectors and Localization, Application to Sheaf Theory, Lec. Notes in Pure Appl. Math. 41, M. Dekker, 1979.

[91] F. van Oystaeyen and L. Willaert, Grothendieck topology, coherent sheaves and Serre's theorem for schematic algebras, J. Pure Appl. Alg. 104 (1995), 109-122.

[92] F. van Oystaeyen and L. Willaert, Cohomology of schematic algebras, J. Alg. 185 (1996), $74-84$

[93] C. Weibel, Homological Algebra, Cambridge Studies in Adv. Math. 38, Cambridge Univ. Press, 1994.

[94] R. Wisbauer, Module and comodule categories - a survey, in: Proc. of the Math. Conf. Birzeit/Nablus, 1998, World Scientific, 2000, 277-304. 